\title{
Influence of grid aspect ratio on planetary boundary layer turbulence in large-eddy simulations
}

\author{
S. Nishizawa, H. Yashiro, Y. Sato, Y. Miyamoto, and H. Tomita \\ RIKEN Advanced Institute for Computational Science, Kobe, Japan \\ Correspondence to: S. Nishizawa (s-nishizawa@riken.jp)
}

Received: 18 May 2015 - Published in Geosci. Model Dev. Discuss.: 4 August 2015

Revised: 15 October 2015 - Accepted: 18 October 2015 - Published: 28 October 2015

\begin{abstract}
We examine the influence of the grid aspect ratio of horizontal to vertical grid spacing on turbulence in the planetary boundary layer (PBL) in a large-eddy simulation (LES). In order to clarify and distinguish them from other artificial effects caused by numerical schemes, we used a fully compressible meteorological LES model with a fully explicit scheme of temporal integration. The influences are investigated with a series of sensitivity tests with parameter sweeps of spatial resolution and grid aspect ratio. We confirmed that the mixing length of the eddy viscosity and diffusion due to sub-grid-scale turbulence plays an essential role in reproducing the theoretical $-5 / 3$ slope of the energy spectrum. If we define the filter length in LES modeling based on consideration of the numerical scheme, and introduce a corrective factor for the grid aspect ratio into the mixing length, the theoretical slope of the energy spectrum can be obtained; otherwise, spurious energy piling appears at high wave numbers. We also found that the grid aspect ratio has influence on the turbulent statistics, especially the skewness of the vertical velocity near the top of the PBL, which becomes spuriously large with large aspect ratio, even if a reasonable spectrum is obtained.
\end{abstract}

\section{Introduction}

In meteorological simulations, the grid aspect ratio $a$ of horizontal $\Delta x$ to vertical grid spacing $\Delta z$ is generally much larger than that in other fluid dynamics fields. Here, we define the aspect ratio as $a=\Delta x / \Delta z$. The use of such large aspect ratios has been validated based on the phenomena studied in this field. So far, most of the phenomena of interest are considerably affected by the rotation of the earth and vertical stratification; both lead to differences between the horizontal and vertical scales of atmospheric phenomena. Further, since it is natural to treat the atmospheric motions separately in the horizontal and vertical directions in order to understand the atmospheric dynamics, a large aspect ratio is used for grids in meteorological simulations. We will have a brief review of the atmospheric phenomena of various scales and the grid aspect ratio used in numerical simulations for the phenomena in the previous studies. This background would help us to understand the grid configuration in current meteorological large-eddy simulations (LESs) and the importance of the investigation of the effect of the grid aspect ratio on the turbulence in LESs.

Atmospheric phenomena have a wide range in terms of both time and space. One of the initial targets of the studies involving meteorological numerical simulations was the planetary wave. In this case, the horizontal scale, at $O(1000)-O(10000) \mathrm{km}$, is much larger than the vertical, and is dominated by the effect of rotation. The vertical scale is mainly determined by atmospheric thickness, characterized by the depth of the troposphere, i.e., a scale of $O(10) \mathrm{km}$, and the motion is essentially two-dimensional (barotropic). The synoptic-scale phenomena, whose horizontal scales are $O(100)-O(1000) \mathrm{km}$, are also important targets, with smaller horizontal scale than planetary-scale phenomena. They have the same vertical scale of $O(10) \mathrm{km}$ as planetary-scale phenomena, but have a vertical (baroclinic) structure. To express the vertical structure of such phenomena, several vertical layers in a numerical simulation are configured according to the degree of vertical structure desired. A grid aspect ratio of $O(100)$ is typically used for these phenomena. 
If we turn our attention to mesoscale phenomena, whose horizontal scales are $O(10)-O(100) \mathrm{km}$, a more detailed expression of vertical structure is required. Since moist convection plays an important role in the vertical motion of such phenomena, hydrostatic balance, which is a good approximation for planetary and synoptic-scale phenomena, is no longer satisfied. The spatial scale of their vertical motion is roughly $O(1) \mathrm{km}$. Therefore, the grid aspect ratio in this case should be reasonably determined with $O(10)-O(100)$. In the beginning of the 1970s, one of the most significant paradigm changes in meteorological numerical studies was the appearance of non-hydrostatic models (e.g., Klemp and Wilhelmson, 1978). Cumulus convection is explicitly represented in these models (e.g., Skamarock et al., 2008; Hodur, 1997; Tripoli and Cotton, 1982; Xue et al., 2000; Black, 1994; Saito et al., 2006; Tsuboki and Sakakibara, 2002). Recently, global convection system-resolving models, such as NICAM (Nonhydrostatic ICosahedral Atmospheric Model; Tomita and Satoh, 2004; Satoh et al., 2014), have been developed to investigate the interactions between synoptic-scale phenomena, cloud clusters, and individual cumulus clouds. To resolve vertical motion precisely, the number of vertical layers in such models is larger than in general circulation models (GCMs), but the grid aspect ratio is still large. For example, it is five in Miyamoto et al. (2013, 2015).

In the atmosphere, there are many smaller scale variabilities than associated with cumulus clouds. For example, the spatial scale of the dominant motion in the unstable planetary boundary layer (PBL) is smaller than $1 \mathrm{~km}$. Clearly, turbulence in the PBL is an important issue for all meteorological models, because heat and mass transport by turbulence in both the horizontal and vertical directions strongly affect the atmospheric mass and energy balances. In particular, vertical transport is important for atmospheric variability over the PBL. Its effect is parameterized as turbulence models in GCMs and cloud-resolving or permitting models (e.g., Mellor and Yamada, 1982; Nakanishi and Niino, 2004). At this scale, the effects of rotation and stratification are smaller compared with those of advection. Thus, we may say that such variability is essentially isotropic and threedimensional. This means that an isotropic grid, whose grid aspect ratio is unity, would be preferred to reproduce these small variabilities.

LES is a vital tool to explicitly represent such small-scale variabilities. It is designed to resolve turbulence at scales down to the inertial sub-range by parameterizing sub-gridscale (SGS) turbulence based on the theory of energy cascades. LES for meteorological simulations has been used since the 1970s (e.g., Deardorff, 1980; Moeng and Wyngaard, 1988; Sullivan et al., 1994), and it is recently used for realistic meteorological simulations with the rapid development of computers (e.g., vanZanten et al., 2011). The dynamics of three-dimensional isotropic turbulence forms the theoretical basis of most SGS models in LESs. In terms of phenomenology and methodology, an isotropic grid would be the most suitable for LES. Nevertheless, the grid used in meteorological LESs usually has a large aspect ratio, mainly because of limitations of computational resources. The computational domain for the meteorological LES has often a wider horizontal range than the vertical range; this is true for the previous meteorological simulations as well: e.g., the domain size has $3 \mathrm{~km}^{2}$ of horizontal region and is $1 \mathrm{~km}$ high in Sullivan et al. (1994), and it has $12.8 \mathrm{~km}^{2}$ of horizontal region and is $4 \mathrm{~km}$ high in vanZanten et al. (2011). A grid with a large aspect ratio reduces the total number of grids. The effect of the grid aspect ratio has not been discussed explicitly in most previous studies. The purpose of this study is to examine the influences of the grid aspect ratio on the turbulent aspects of the PBL from a meteorological viewpoint when LES is applied. To do so, we performed a series of PBL experiments by systematic parameter sweeps of aspect ratio and grid spacing.

We present an overview of the model used in this study in Sect. 2, focusing on the modeling related to this study. Since the detailed descriptions of the model are important for the traceability of the experiment and reliability of the model, we present them in the appendices. The configuration of the numerical experiments is shown in Sect. 3. The influences of the grid aspect ratio on turbulence are discussed in Sect. 4. Section 5 gives the summary and concluding remarks.

\section{Model description}

The model used in this study is SCALE-LES (Scalable Computing for Advanced Library and Environment-LES; http: //scale.aics.riken.jp/), which was developed as a meteorological large-eddy simulation model. In this section, we focus on describing the essential parts of the model for the purposes of this study; the model dynamics and turbulence schemes are described in Sect. 2.1 and 2.2, respectively. In the latter, we consider in particular the relationship between the numerical filter and spatial filter in LES.

Detailed descriptions of the model, including the discretization method, which are not directly related to the topic of this study, are given in the appendices as follows: the governing equations are in Appendix A1, the temporal integration scheme is in Appendix A2, the spatial discretization is in Appendix A3, the numerical filter is in Appendix A4, and other physical processes are in Appendix A5. We also describe the consideration of numerical stability in determining discretization schemes in Appendix B, and the validity of the dynamical core in Appendix C. Symbols used in this paper are summarized in Table 1.

\subsection{Dynamics}

Since the purpose of this study is to clarify the impact of the grid aspect ratio on the turbulent aspects, the influences of the approximations to the governing equations should be 
Table 1. Notation of symbols.

\begin{tabular}{|c|c|c|}
\hline$\rho$ & total density & $\mathrm{kg} \mathrm{m}^{-3}$ \\
\hline$q_{\mathrm{d}}$ & ratio of mass of dry air to total mass & - \\
\hline$q_{\mathrm{V}}$ & specific humidity & - \\
\hline$q_{1}$ & specific liquid water content & - \\
\hline$q_{\mathrm{s}}$ & specific solid water content & - \\
\hline$t$ & time & $\mathrm{s}$ \\
\hline $\boldsymbol{u}$ & velocity vector of air flow & $\mathrm{m} \mathrm{s}^{-1}$ \\
\hline$u$ & $x$ component of velocity & $\mathrm{ms}^{-1}$ \\
\hline$v$ & $y$ component of velocity & $\mathrm{ms}^{-1}$ \\
\hline$w$ & $z$ component of velocity & $\mathrm{ms}^{-1}$ \\
\hline$w_{1}$ & relative velocity of liquid water to gas & $\mathrm{ms}^{-1}$ \\
\hline$w_{\mathrm{s}}$ & relative velocity of solid water to gas & $\mathrm{ms} \mathrm{s}^{-1}$ \\
\hline$S_{\mathrm{V}}$ & source term of water vapor & $\mathrm{kg} \mathrm{m}^{-3} \mathrm{~s}^{-1}$ \\
\hline$S_{1}$ & source term of liquid water & $\mathrm{kg} \mathrm{m}^{-3} \mathrm{~s}^{-1}$ \\
\hline$S_{\mathrm{S}}$ & source term of solid water & $\mathrm{kg} \mathrm{m}^{-3} \mathrm{~s}^{-1}$ \\
\hline$p$ & pressure & $\mathrm{Nm}^{-2}$ \\
\hline$g$ & gravitational acceleration & $\mathrm{ms} \mathrm{s}^{-2}$ \\
\hline$f_{1}$ & drag force from water loading by liquid water & $\mathrm{kg} \mathrm{m}^{-2} \mathrm{~s}^{-2}$ \\
\hline$f_{\mathrm{S}}$ & drag force from water loading by solid water & $\mathrm{kg} \mathrm{m}^{-2} \mathrm{~s}^{-2}$ \\
\hline $\boldsymbol{e}_{z}$ & vertical unit vector (upward) & - \\
\hline$R_{\mathrm{d}}$ & gas constant for dry air for unit mass & $\mathrm{J} \mathrm{kg}^{-1}$ \\
\hline$R_{\mathrm{V}}$ & gas constant for water vapor for unit mass & $\mathrm{J} \mathrm{kg}^{-1}$ \\
\hline$T$ & temperature & $\mathrm{K}$ \\
\hline$Q_{\mathrm{d}}$ & diabatic heating from dry air & $\mathrm{J} \mathrm{m}^{-3} \mathrm{~s}^{-1}$ \\
\hline$Q_{\mathrm{V}}$ & diabatic heating from water vapor & $\mathrm{J} \mathrm{m}^{-3} \mathrm{~s}^{-1}$ \\
\hline$Q_{1}$ & diabatic heating from liquid water & $\mathrm{J} \mathrm{m}^{-3} \mathrm{~s}^{-1}$ \\
\hline$Q_{\mathrm{S}}$ & diabatic heating from solid water & $\mathrm{J} \mathrm{m}^{-3} \mathrm{~s}^{-1}$ \\
\hline$e_{\mathrm{d}}$ & internal energy of dry air & $\mathrm{J} \mathrm{kg}^{-1}$ \\
\hline$e_{\mathrm{V}}$ & internal energy of water vapor & $\mathrm{J} \mathrm{kg}^{-1}$ \\
\hline$e_{1}$ & internal energy of liquid water & $\mathrm{J} \mathrm{kg}^{-1}$ \\
\hline$e_{\mathrm{S}}$ & internal energy of solid water & $\mathrm{J} \mathrm{kg}^{-1}$ \\
\hline$e$ & total internal energy & $\mathrm{J} \mathrm{kg}^{-1}$ \\
\hline$c_{v \mathrm{~d}}$ & specific heat at constant volume of dry air & $\mathrm{J} \mathrm{kg}^{-1} \mathrm{~K}^{-1}$ \\
\hline$c_{v \mathrm{v}}$ & specific heat at constant volume of water vapor & $\mathrm{J} \mathrm{kg}^{-1} \mathrm{~K}^{-1}$ \\
\hline$c_{p \mathrm{~d}}$ & specific heat at constant pressure of dry air & $\mathrm{J} \mathrm{kg}^{-1} \mathrm{~K}^{-1}$ \\
\hline$c_{p \vee}$ & specific heat at constant pressure of water vapor & $\mathrm{J} \mathrm{kg}^{-1} \mathrm{~K}^{-1}$ \\
\hline$c_{1}$ & specific heat of liquid water & $\mathrm{J} \mathrm{kg}^{-1} \mathrm{~K}^{-1}$ \\
\hline$c_{\mathrm{S}}$ & specific heat of solid water & $\mathrm{J} \mathrm{kg}^{-1} \mathrm{~K}^{-1}$ \\
\hline$p_{00}$ & standard pressure & $\mathrm{Pa}$ \\
\hline$\theta_{\mathrm{d}}$ & potential temperature of dry air & $\mathrm{K}$ \\
\hline$\theta$ & potential temperature of total air & $\mathrm{K}$ \\
\hline
\end{tabular}

reduced as much as possible. For this reason, we employ the set of fully compressible non-hydrostatic equations as the governing equations. The three-dimensional momentum ( $\rho u, \rho v$, and $\rho w$, for the $x, y$, and $z$ directions, respectively), total density $(\rho)$, mass-weighted potential temperature $(\rho \theta)$, and mass concentration of tracers $\left(\rho q_{x} ; x \in\{v, l, s\}\right)$ are used as the prognostic variables. See Appendix A1 for the detailed formulation of the governing equations.

The central difference schemes are used for spatial discretization; the second-order scheme is applied to the pressure gradient terms in the momentum equations and divergence terms of mass flux in the continuity equation, while the fourth-order scheme is applied to the advection terms in the momentum and thermodynamics equations. The reason the advection terms are discretized by the high-order scheme is based on the accuracy of the eddy viscosity and the diffusion terms representing the effect of SGS turbulence. The coefficients of the viscosity and diffusion terms are proportional to the square of the grid spacing, so that the magnitude of the terms would be comparable to the truncation error of the advection terms, in terms of order of accuracy, if the second-order scheme is employed. Additionally, the higher-order treatment for the advection terms is necessary from a different viewpoint as well. Since the advective term is a non-linear convolution, it requires a higher-order treatment to resolve the additional modes. The use of the lowerorder scheme is justified by the scale separation of the fast modes (acoustic and fast gravity waves) and slow modes (advection). In the meteorological phenomena, the terms of the pressure gradient in the momentum equations and the divergence in the continuity equation are dominant for the fast modes, while the advection term is dominant for the slow modes. The interaction between the fast and slow modes is not significant generally. If we consider SGS mixing in a local field such as a several-grid scale, the fast waves would pass over this field soon before completing the SGS mixing. This means that fast waves do not participate much in the local mixing, compared with the mixing process itself.

The even-order schemes require the explicit numerical filter for numerical stability. However, they have the advantage that it is easier to evaluate the effect of the numerical diffusivity using the explicit numerical filter than that introduced implicitly by an odd-order scheme. See Appendices A3 and A4 for details of the discretization and a discussion of the numerical filter, respectively.

A fully explicit scheme, i.e., HE-VE (horizontally explicit and vertically explicit) scheme, is used for temporal integration in this study. This scheme generally has less implicit numerical diffusion than implicit schemes such as HI-VI (horizontally implicit and vertically implicit) and HE-VI (horizontally explicit and vertically implicit) schemes. To focus on the influences of the grid aspect ratio, an explicit scheme is more suitable than implicit schemes. Additionally, we do not use a time-split scheme to avoid numerical damping of the time splitting. See Appendix A2 for a detailed description of the temporal scheme used in this study.

In order to validate the dynamical core of this model, we performed a density current experiment (Straka et al., 1993) as a standard test case. The detailed results are described in Appendix C; we posit that the dynamical core of the model shows reasonable performance, and is reliable enough for the investigations performed in this study. As well as validating the dynamical core, Sato et al. (2015) validated this model from the viewpoint of moist physical processes. 


\subsection{Sub-grid model}

\subsubsection{Overview of LES modeling}

The model for effect of SGS turbulence used in this model is a Smagorinsky-Lilly-type model (Smagorinsky, 1963; Lilly, 1962). In the governing equations shown in Appendix A1, the effect of the SGS turbulence can be written as $\frac{\partial \rho \tau_{i j}}{\partial x_{j}}$ in the momentum equations, and $\frac{\partial \rho \tau_{i}^{*}}{\partial x_{i}}$ in the thermodynamics and tracer equations. In this subsection, the subscripts imply summation over the set $\{1,2,3\}$. The $\tau_{i j}$ and $\tau_{i}^{*}$ are parameterized as

$\tau_{i j}=-2 \nu_{\mathrm{SGS}}\left(S_{i j}-\frac{1}{3} S_{k k} \delta_{i j}\right)+\frac{2}{3} \mathrm{TKE}_{\mathrm{SGS}} \delta_{i j}$,

$\tau_{i}^{*}=-v_{\mathrm{SGS}}^{*} \frac{\partial \phi}{\partial x_{i}}$,

where $\nu_{\mathrm{SGS}}$ and $\nu_{\mathrm{SGS}}^{*}$ are the coefficients of the SGS eddy viscosity and diffusion, respectively, $\delta_{i j}$ is the Kronecker delta, and $\phi$ represents scalar quantities, such as $\theta$ and $q_{x} . S$ is the strain tensor given by

$S_{i j}=\frac{1}{2}\left(\frac{\partial u_{i}}{\partial x_{j}}+\frac{\partial u_{j}}{\partial x_{i}}\right)$.

$\mathrm{TKE}_{\mathrm{SGS}}$ is the turbulence kinetic energy (TKE) of the SGS turbulence:

$\mathrm{TKE}_{\mathrm{SGS}}=\frac{1}{2} \tau_{i i}$

The second term on the right-hand side of Eq. (1) arose because of the consistency with Eq. (4) in the compressive flow (e.g., Moin et al., 1991).

The role of the sub-grid model is to parameterize the effect of SGS turbulence based on the energy cascade theory of three-dimensional isotropic turbulence. The eddy viscosity and diffusion model is employed as a sub-grid model to represent the effect. For the determination of the amount of the energy cascade, the mixing length of the eddy viscosity and diffusion is the most important factor. The coefficient of the SGS eddy viscosity, $v_{\text {SGS }}$ in Eq. (1), is proportional to the square of the mixing length $\left(L_{\text {mix }}\right)$ in Prandtl's mixing length theory:

$v_{\mathrm{SGS}}=L_{\text {mix }}^{2}|S|$.

The mixing length depends on what type of spatial filter we employ on the variables in the equations, and the length scale of the spatial filter is the essential factor for the mixing length. The spatial filtering is inevitable in the discretization of the equations. The spatial filter in a numerical model is implicitly determined by the grid spacing and discretization schemes. The artificial length characterizing the spatial filter owing to discretization is defined as the filter length. Besides the filter length, the shape of the grid should be also considered in the mixing length determination. Its effect can be represented by the grid aspect ratio. Scotti et al. (1993) proposed an equation for the effect of the grid aspect ratio on the mixing length. Using the filter length $\Delta$ and grid aspect ratio $a$, the mixing length can be represented as

$L_{\text {mix }}=C_{\mathrm{s}} f(a) \Delta F_{\mathrm{S}}$,

where $f(a)$ is a function of the grid aspect ratio, and represents the effect of the grid aspect ratio on the mixing length. $C_{\mathrm{S}}$ is the Smagorinsky constant, and $F_{\mathrm{S}}$ represents the effect of static stability.

The effect of the static stability on the mixing length is introduced in this model according to Brown et al. (1994). They extended the Lilly model to improve the performance of the simulation by considering the dependency of the Prandtl number, $\operatorname{Pr}=v_{\mathrm{SGS}} / \nu_{\mathrm{SGS}}^{*}$. For the unstable condition $(R i<0)$,

$\nu_{\mathrm{SGS}}=\left(C_{\mathrm{S}} f(a) \Delta\right)^{2}|S| \sqrt{1-c R i}$,

$\operatorname{Pr}=\operatorname{Pr}_{\mathrm{N}} \sqrt{\frac{1-c R i}{1-b R i}}$,

where $R i$ is the local (pointwise) gradient Richardson number, defined as

$R i=\frac{N^{2}}{|S|^{2}}$.

$P r_{\mathrm{N}}$ is the Prandtl number in neutral conditions; $P r_{\mathrm{N}}=0.7$. The constants $c$ and $b$ are 16 and 40, respectively, following Brown et al. (1994). They are empirically given to fit surfacelayer observations. For the weakly stable condition, where $R i$ is smaller than the critical Richardson number $R i_{\mathrm{c}}(=0.25)$, i.e., $0 \leq R i<R i_{\mathrm{c}}$,

$v_{\mathrm{SGS}}=\left(C_{\mathrm{s}} f(a) \Delta\right)^{2}|S|\left(1-\frac{R i}{R i_{\mathrm{c}}}\right)^{4}$,

$\operatorname{Pr}=\operatorname{Pr}_{\mathrm{N}}\left\{1-\left(1-\operatorname{Pr}_{\mathrm{N}}\right) \frac{R i}{R i_{\mathrm{c}}}\right\}^{-1}$.

For the strongly stable condition $\left(R i \geq R i_{\mathrm{c}}\right)$,

$v_{\mathrm{SGS}}=0$,

$\operatorname{Pr}=1$.

The TKE $E_{S G S}$ in Eq. (1) is

$\mathrm{TKE}_{\mathrm{SGS}}=\left(\frac{\nu_{\mathrm{SGS}}}{C_{k} \Delta}\right)^{2}$

where $C_{k}$ is an SGS constant and assumed to be 0.1 , following Deardorff (1980) and Moeng and Wyngaard (1988). 


\subsubsection{Problems of filter length and grid aspect ratio}

There are two ambiguous factors in the configuration for determining the mixing length in recent meteorological LES models. One problem is the configuration of the filter length in Eq. (6) in the models. The value of $\Delta$ is usually set as the grid spacing for simplicity, not by considering the numerical filter inherited by an individual model scheme. The other problem is that the effect of the grid aspect ratio on the mixing length is not considered in determining the mixing length; correspondingly, $f(a)=1$ in Eq. (6).

Such rough treatment of the filter length and no consideration of the grid aspect ratio lead to incorrect turbulent properties. A usual remedy for such effects is a posteriori tuning of the Smagorinsky constant, $C_{\mathrm{s}}$ in Eq. (6), so as to reproduce realistic results (e.g., Deardorff, 1971). However, this constant should not, in principle, be determined empirically, but should be derived from the theory. Some procedures, called dynamic SGS models, derive the constant dynamically (e.g., Germano et al., 1991). They are advantageous in the case in which the assumption of the isotropic turbulence is not justified, e.g., very close to a boundary. However, in our opinion, dynamic SGS models are associated with some practical problems, e.g., numerical stability; more importantly, they seem to be a type of a mathematical procedure and do not seem to have a physical basis.

Here, we should note that each theory is based on its own basic concepts. Although this means that different theoretical concepts lead to different values for the constants, we should keep in mind that a certain constant is uniquely determined by a particular model on a theoretical basis. The Smagorinsky constant is derived by integration of the kinetic energy filtered out by the spatial filter. If the cutoff filter is employed as the spatial filter, the integration can be performed in the cubic $B$ in Fourier space:

$B=\left\{\left|k_{x}\right|<\pi / \Delta x,\left|k_{y}\right|<\pi / \Delta y,\left|k_{z}\right|<\pi / \Delta z\right\}$,

where $k_{i}$ is the wave number in the $i$-direction. Lilly (1967) obtained the constant $\left(C_{\mathrm{s}}=0.16\right)$ by integrating in the sphere inscribed in the cubic $B$, while Scotti et al. (1993) obtained it more precisely by integrating in the entire cubic. In this study, we follow Scotti et al. (1993), and use $C_{\mathrm{s}}=0.13$.

Let us return to the first problem, i.e., the determination of the spatial filter length $\Delta$. Most models in previous studies assume that the filter length is determined as the geometric mean of the grid spacings in the three directions (e.g., Deardorff, 1980):

$\Delta=(\Delta x \Delta y \Delta z)^{1 / 3}$.

It is generally difficult to strictly define the spatial filter in a model because the filtering effect implicitly introduced by discretization is very complicated. This difficulty is especially significant if we apply the implicit method of time integration. On the other hand, the effect of the explicitly introduced numerical filter can be more easily estimated than that of an implicitly introduced filter. In our model, we introduce hyperviscosity and diffusion explicitly as the numerical filter (see Appendix A4). The explicit numerical filter is thought to be the dominant spatial filter because we employ the explicit temporal integration method and even-order spatial difference scheme. This type of filter removes the two-grid-scale noise selectively. In other words, the two-grid-scale variability is filtered out from the resolved variability. Consequently, we can say that in our model, the two-grid scale is preferred as the filter length rather than the grid spacing defined in Eq. (16):

$\Delta=2(\Delta x \Delta y \Delta z)^{1 / 3}$.

In the next section, we investigate the validity of this configuration using simulation results.

The second problem is the treatment of the grid aspect ratio in LES in actual grid systems. In the equilibrium condition with the universal Kolmogorov spectrum, the energy flux cascaded into the SGS variability is equal to the SGS dissipation. Since the dissipation does not depend on the artificial grid configuration but on the physical configuration, energy cascaded to the SGS turbulence should not depend on grid configuration, including the grid aspect ratio. The energy cascade flux, which is equal to the dissipation, can be written as a function of the strain tensor $S_{i j}$ defined in Eq. (3) and the mixing length in Eq. (6) in Smagorinsky-type models. $S_{i j}$ depends on the grid configuration, including aspect ratio. The mixing length should be thus dependent on the aspect ratio to cancel out the dependency of $S_{i j}$ on the aspect ratio; otherwise, the energy cascade flux and dissipation should be dependent on the grid configuration. Scotti et al. (1993) derived an approximate function of the $f(a)$ in Eq. (6), considering the grid aspect ratio theoretically by integrating the energy in the cubic $B$ defined in Eq. (15):

$$
\begin{aligned}
f(a)= & 1.736 a^{1 / 3}\left\{4 P_{1}\left(b_{1}\right) a^{1 / 3}+0.222 P_{2}\left(b_{1}\right) a^{-5 / 3}\right. \\
& +0.077 P_{3}\left(b_{1}\right) a^{-11 / 3}-3 b_{1}+4 P_{1}\left(b_{2}\right) \\
& \left.+0.222 P_{2}\left(b_{2}\right)+0.077 P_{3}\left(b_{2}\right)-3 b_{2}\right\}^{-3 / 4},
\end{aligned}
$$

where $b_{1}=\arctan (1 / a), b_{2}=\arctan (a)=\pi / 2-b_{1}$, and

$$
\begin{aligned}
P_{1}(z)= & 2.5 P_{2}(z)-1.5(\cos (z))^{2 / 3} \sin (z), \\
P_{2}(z)= & 0.98 z+0.073 z^{2}-0.418 z^{3}+0.120 z^{4}, \\
P_{3}(z)= & 0.976 z+0.188 z^{2}+1.169 z^{3} \\
& +0.755 z^{4}-0.151 z^{5} .
\end{aligned}
$$

Note that they considered two grid aspect ratios, while here we consider only one for simplicity, i.e., $\Delta x=\Delta y>$ $\Delta z$. Note that $f(a)$ is a monotonic function for $a$, for instance, $f(2)=1.036, f(5)=1.231, f(10)=1.469$, and $f(20)=1.790$. Roughly speaking, there can exist larger scale (or lower wave number) variability in filtered out variabilities for a larger grid aspect ratio. This implies larger 
Table 2. Filter lengths and the effect of grid aspect ratio on mixing length in the three experiments performed in this study.

\begin{tabular}{lll}
\hline & Filter length & $\begin{array}{l}\text { Modification by } \\
\text { the grid aspect ratio }\end{array}$ \\
\hline Control experiment & $2(\Delta x \Delta y \Delta z)^{1 / 3}$ & yes \\
Small filter length experiment & $(\Delta x \Delta y \Delta z)^{1 / 3}$ & yes \\
Fixed mixing length experiment & $2(\Delta x \Delta y \Delta z)^{1 / 3}$ & no $(f(a)=1)$ \\
\hline
\end{tabular}

turbulence kinetic energy for larger aspect ratios, and larger TKE results in a larger mixing length. In our study, the effect of the grid aspect ratio on the mixing length is introduced in Eq. (18) by following Scotti et al. (1993).

\section{Numerical experiments}

We performed three PBL experiments to examine the influences of the grid aspect ratio and filter length on simulated turbulence, which are summarized in Table 2. In the control experiment, the filter length is double the grid spacing, as in Eq. (17), and the mixing length in the sub-grid model is modified by the grid aspect ratio, as in Eq. (18). The second experiment is a small filter length experiment in which the filter length is set as in Eq. (16). The last is a fixed mixing length experiment, in which the mixing length in the SGS model is not modified by the grid aspect ratio; that is, $f(a)=1$. In the most current meteorological simulations, the configuration of the filter length follows Eq. (16) and $f(a)=1$ in Eq. (6).

In the three experiments above, a systematic parameter sweep of resolution and grid aspect ratios was conducted. The spatial resolution of each run performed in the control experiment is shown in Table 3. The parameter set in the small filter length experiment is the same as the control experiment, except for the 5mAR10 and 5mAR20 runs, while in the fixed mixing length experiment it is the same as the control, except for the 10mAR3 run.

In all the runs, the domain size is $9.6 \mathrm{~km} \times 9.6 \mathrm{~km}$ in the horizontal direction, and $3 \mathrm{~km}$ in the vertical. The domain size is large enough to contain convective cells in the PBL. The lateral boundary conditions are double periodic. The vertical grid is stretched above $2 \mathrm{~km}$. In the stretching layer, the vertical velocity is damped down by Rayleigh damping with an $e$-folding time of $10 \mathrm{~s}$ to reduce the effect of the reflection of waves at the top boundary.

For the initial condition, we follow Ito et al. (2010); the potential temperature is $299 \mathrm{~K}$ at the surface, with a vertical gradient of $-4 \mathrm{~K} \mathrm{~km}^{-1}$ and a random small perturbation of $0.1 \mathrm{~K}$. A constant horizontal velocity of $5 \mathrm{~m} \mathrm{~s}^{-1}$ is set in the $x$ direction in the entire domain.

The temporal integration is done for 4 hours for each run. In order to focus on our purpose, we simplify the setting of the bottom boundary condition so that a constant heat flux of $200 \mathrm{~W} \mathrm{~m}^{-2}$ is added to the lowest layer through the in-
Table 3. Names of runs we performed in the control experiment. Columns and rows correspond to vertical and horizontal resolutions, respectively. The number following the character "AR" represents the grid aspect ratio.

\begin{tabular}{llll}
\hline$\Delta x \Delta z$ & $5 \mathrm{~m}$ & $10 \mathrm{~m}$ & $30 \mathrm{~m}$ \\
\hline $10 \mathrm{~m}$ & - & $10 \mathrm{mAR} 1$ & - \\
$20 \mathrm{~m}$ & - & $10 \mathrm{mAR} 2$ & - \\
$30 \mathrm{~m}$ & - & $10 \mathrm{mAR} 3$ & $30 \mathrm{mAR} 1$ \\
$50 \mathrm{~m}$ & $5 \mathrm{mAR} 10$ & $10 \mathrm{mAR} 5$ & - \\
$60 \mathrm{~m}$ & - & $10 \mathrm{mAR} 6$ & $30 \mathrm{mAR} 2$ \\
$100 \mathrm{~m}$ & $5 \mathrm{mAR} 20$ & $10 \mathrm{mAR} 10$ & - \\
$150 \mathrm{~m}$ & - & - & $30 \mathrm{mAR} 5$ \\
\hline
\end{tabular}

tegration, although Ito et al. (2010) added a heat flux with a sinusoidal temporal change to represent diurnal change. With a constant flow of $5 \mathrm{~m} \mathrm{~s}^{-1}$ and a ground heat flux of $200 \mathrm{~W} \mathrm{~m}^{-2}$, we expect the cellular convection to develop according to Ito et al. (2010). In this study, we consider dry conditions without moist and radiation processes.

Different temporal intervals are used for the dynamical and physical processes. We define the dynamical process as that related to fluid dynamics; the advection, pressure gradient, and gravitational force terms in the governing equations are treated as dynamical processes in this model. The other processes are physical processes in this model. In this study, the physical processes are only the surface flux for the momentum and the eddy viscosity and diffusion for SGS turbulence. Note that we treat the eddy viscosity and diffusion terms, which originated from the advection term, as the physical process for correspondence with the sub-grid turbulence model in RANS (Raynolds-averaged Navier-Stokes) mode in this model. The intervals for the dynamical process $\Delta t_{\text {dyn }}$ are $0.006,0.012$, and $0.03 \mathrm{~s}$ for $\Delta z=5,10$, and $30 \mathrm{~m}$, respectively. The interval for the physical processes $\Delta t_{\text {phys }}$ is 10 times $\Delta t_{\text {dyn }}$ to reduce computational resource. One concern is that use of too large a factor of $\Delta t_{\text {phys }} / \Delta t_{\text {dyn }}$ has the non-negligible effect of an artificial acoustic wave excited by intermittent forcing added by the physical processes. In this model, the artificial wave can be reduced as described in Appendix A2.2. In a preliminary experiment, we confirmed that simulated phenomena in the runs with $\Delta t_{\text {phys }}=\Delta t_{\text {dyn }}$ and $\Delta t_{\text {phys }}=10 \Delta t_{\text {dyn }}$ do not have essential differences in the context of this study.

We used an eighth-order numerical filter, i.e., $n=8$ in Eq. (A125), with the non-dimensional coefficient $\gamma$ of $2 \times$ $10^{-4}$ in this study. The higher order is preferred in order to limit the numerical filter to smaller scale variability, but the higher-order filter requires more expensive computational costs. As discussed in Appendix A4, we confirmed that the effect of the numerical filter of order eight is less than that of the eddy diffusion and viscosity. The smaller coefficient $\gamma$ is preferred as long as two-grid scale noise is prevented. The coefficient was determined by another preliminary parameter 
sweep experiment of the coefficient without the SGS model for the numerical filter to prevent two-grid noise.

\section{Results and discussions}

\subsection{Energy spectrum}

Most sub-grid models are based on the idea of an energy cascade due to three-dimensional isotropic turbulence so that the energy spectrum has the slope of $k^{-5 / 3}$, where $k$ is the wave number. Figure 1a shows the horizontal spectrum of the three-dimensional kinetic energy at an altitude of $500 \mathrm{~m}$ in the control experiment. This height corresponds to the middle of the PBL, at which the vertical velocity is largest. The inertial sub-range with $-5 / 3$ power can be clearly seen at wave numbers greater than $1 / 1000 \mathrm{~m}^{-1}$. The spectra for runs with various resolutions and grid aspect ratios show good agreement with one another, except near the filter length scale, at which variabilities are damped down numerically. The largest energy appears at a scale of about $2 \mathrm{~km}$; the external forcing seems to mainly inject energy at this scale. This corresponds to the spatial structure of convective cells, as shown in Fig. 3a. The cells have well-known hexagonal or quadrangular structure with strong upward convection in the narrow cell boundary, and relatively weak downward convection in the entire cell region.

On the other hand, the spectrum of horizontal kinetic energy $u^{2}+v^{2} / 2$ does not clearly indicate the power law as shown in Fig. 2. Horizontal vortexes (i.e., convections) are strongly constrained by the PBL top and bottom boundaries. The horizontal velocity related to convection is dominant near the top and bottom of the convection, while it is relatively small near the middle of the convection. As a result, the horizontal kinetic energy of large-scale motion tends to be smaller around the middle of the PBL. On the other hand, the vertical velocity is dominant in the middle of the convection, and the energy spectrum of vertical kinetic energy mostly follows the power law, as shown in Fig. 2. This difference between vertical and horizontal velocity is more significant for larger scale motion. This is almost opposite for horizontal and vertical velocities near the PBL top and bottom boundaries; the spectrum of the horizontal velocity at the lowest layer shows a slope of almost $-5 / 3$, while that of the vertical velocity does not. These differences, due to the existence of the PBL top and bottom boundaries, cause anisotropy as well as static stability (Yakhot et al., 1989; Horiuti, 1993).

In most LES models, the filter length for the sub-grid model is set to be equal to the grid spacing itself. In our model, the filter length is set to be twice the grid spacing, as described in Sect. 2. In order to examine the influence of the filter length, we performed the small filter length experiment, in which we use a conventional filter length, i.e., as in Eq. (16). Figure $1 \mathrm{~b}$ shows the energy spectrum in this ex-
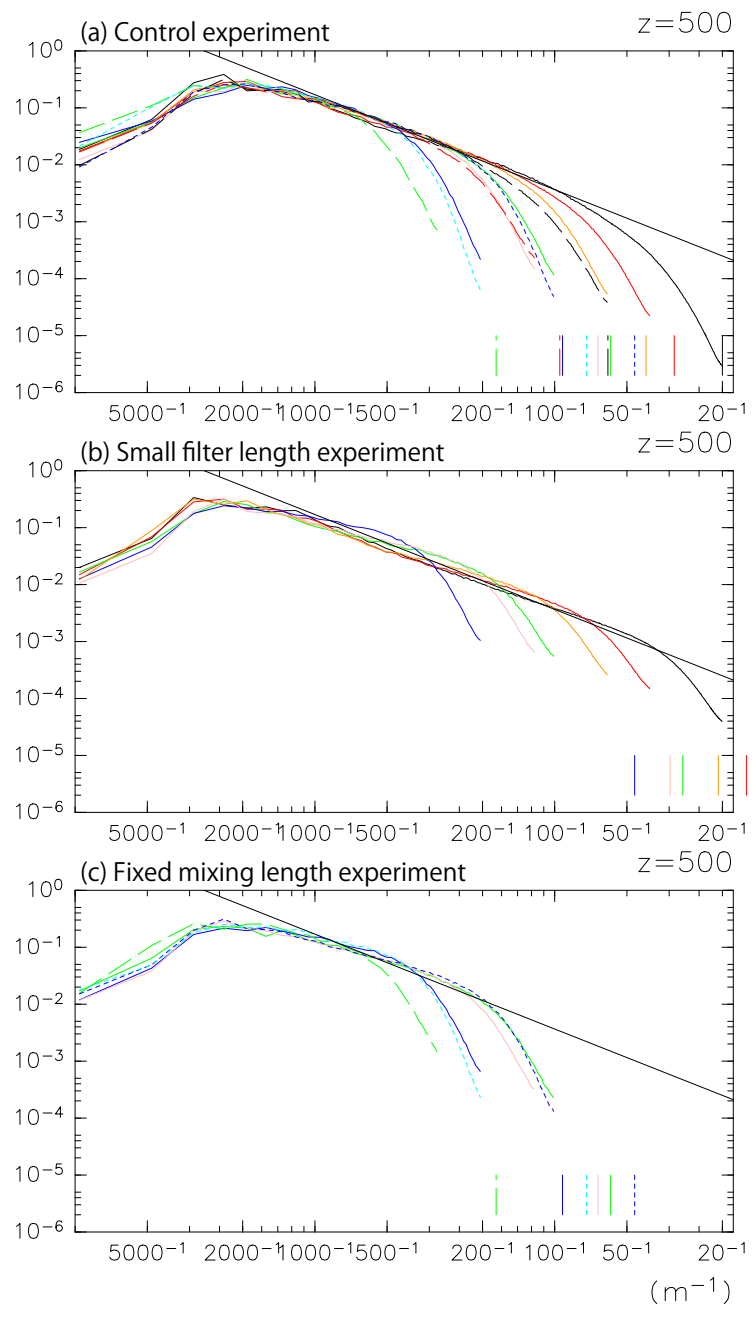

Figure 1. Energy spectrum three-dimensional velocity at height of $500 \mathrm{~m}$ averaged over $t=3.5$ to $4 \mathrm{~h}$ in (a)control experiment, (b) small filter length experiment, and (c) fixed mixing length experiment. Colors indicate grid aspect ratio: 1 (black), 2 (red), 3 (orange), 5 (green), 6 (pink), 10 (blue), and 20 (cyan). Line types indicate vertical resolution: $10 \mathrm{~m}$ (solid line), $30 \mathrm{~m}$ (dashed line), and $5 \mathrm{~m}$ (dotted line). Linear slope representing the slope of $-5 / 3$ power fit to the spectrum of the $10 \mathrm{mAR} 1$ run between $1 / 1000$ and $1 / 100 \mathrm{~m}^{-1}$ is superimposed. Only spectra for $\Delta z=10 \mathrm{~m}$ runs in the small filter length experiment and for large aspect ratio runs $(a \geq 5)$ in the fixed mixing length experiment are shown.

periment (only runs with $\Delta z=10 \mathrm{~m}$ are shown). The spectra show a spurious energy profile, in which energy piles up at the higher wave numbers. The slopes of the spectra become gentler than $-5 / 3$ because of the spurious energy. The spurious energy piling is considered to be a result of the lack of energy cascade because of SGS variability, caused by the use of a small mixing length. We found that the situation is more significant for larger grid aspect ratios and coarser horizontal resolution, as shown Fig. 1b, although the effect of the grid 

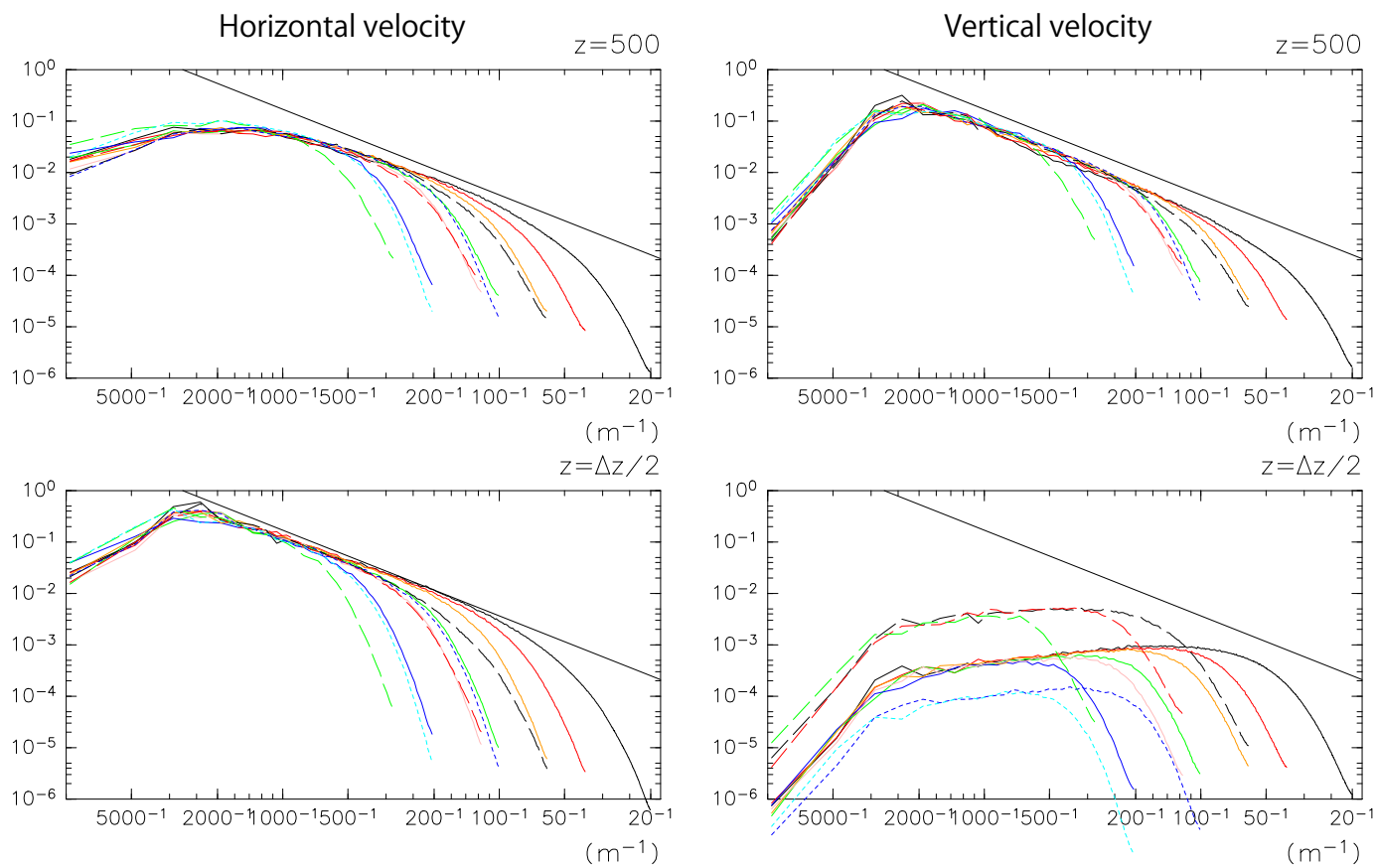

Figure 2. Same as Fig. 1, but spectrum of (left) horizontal and (right) vertical velocity at (top) height of $500 \mathrm{~m}$ and (bottom) lowest layer $(z=2.5,5$, and $15 \mathrm{~m}$ for $\Delta z=5,10$, and $30 \mathrm{~m})$ in the control experiment.

aspect ratio represented by Eq. (18) is taken into account in this experiment, as well as in the control experiment.

The deviation from the theoretical $-5 / 3$ slope of the energy spectrum of resolved variability is more obvious at the energy-dissipative range. However, the reduced energy is compensated by the SGS energy. On the other hand, the energy pile cannot be compensated by any SGS components. Therefore, the spurious energy pile is the most important point in terms of the reproducibility of the spectrum. In order to estimate the degree of the energy piling quantitatively, we introduce an index denoted by SEP (spurious energy pile):

$\mathrm{SEP} \equiv \max \left(\frac{E(k)}{A k^{-5 / 3}}\right)$,

where $E(k)$ is the energy spectrum in each run, and the fitting coefficient $A$ is calculated with a spectrum ranging from $1 / 1000$ to $1 / 100 \mathrm{~m}^{-1}$ using the highest resolution run with a grid aspect ratio of unity (10mAR1 run) in the control experiment as a reference solution. Thus, SEP indicates the maximum ratio of the energy spectrum in each run to the fitting slope of $-5 / 3$ power in the reference run. Note that the index is not influenced any more by the lower energy than the theoretical slope at the energy-containing and energydissipative scales. Figure 4 shows the dependency of the SEP index on the grid aspect ratio. Since the energy spectrum is not perfectly a power law with the $-5 / 3$ slope, the index is not 1 , even in the $10 \mathrm{mAR} 1$ run. The index in the control experiment is smaller than 1.2 for all the runs. On the other hand, the indices in the small filter length experiment are larger than those in the control experiment. It tends to be larger for the larger aspect ratio. This tendency can be found for both the vertical grid resolutions of 10 and $30 \mathrm{~m}$. The magnitude of the SEP is larger than 1.2 for all the runs, except when the grid aspect ratio is unity. This shows that, in this model, twice the grid spacing is more suitable as the filter length rather than the grid spacing itself, and suggests that the filter length should be chosen according to the numerical schemes used in a model. In addition, the dependency on the grid aspect ratio can clearly be seen. These results suggest that an appropriate filter length is necessary, especially in the case of a large grid aspect ratio.

Next, we consider the influence of $f(a)$ in the mixing length, as in Eq. (6), on the spectrum. The mixing length is modified by the grid aspect ratio in the control experiment according to Scotti et al. (1993), as described in Sect. 2. In the fixed mixing length experiment, we fixed $f(a)$ in Eq. (6) equal to 1 in order to ignore the effect of the grid aspect ratio. Figure 1c shows the spectra of the runs in the fixed mixing length experiment; only runs with larger grid aspect ratios are shown. The spectra for the large aspect ratio, especially greater than 10, show the spurious energy pile, as in the case of the small filter length experiment. The SEP index is greater than 1.3 in these cases, as shown in Fig. 4. This means that the effect of the grid aspect ratio should be taken into account in modeling the mixing length for the SGS turbulence model. The mixing length should be enlarged according to the effect of the grid aspect ratio from that of the isotropic grid case; otherwise, artificial energy piles at higher wave numbers. 


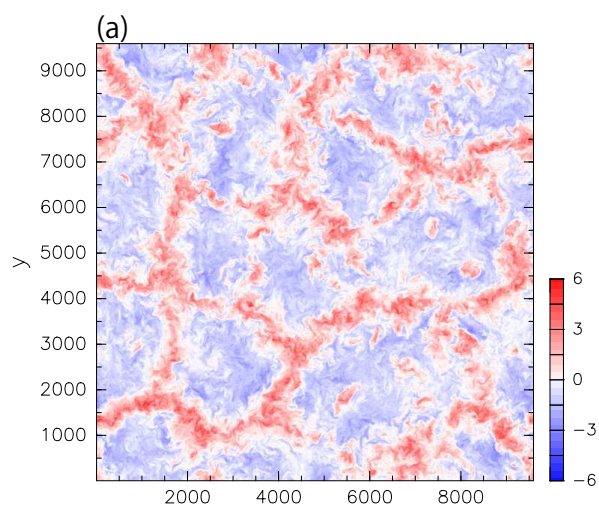

(b)

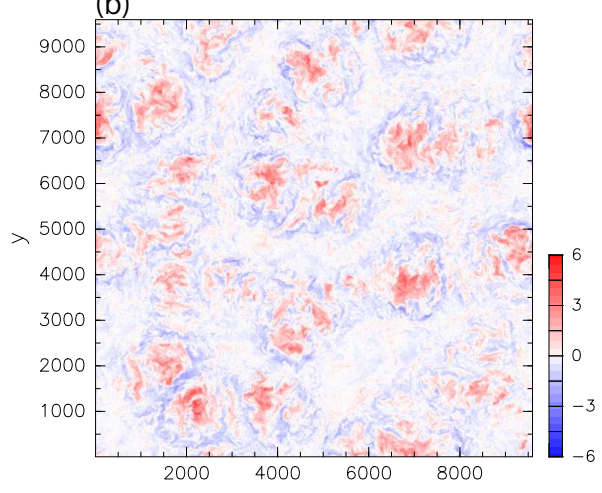

Figure 3. Horizontal cross section of the vertical velocity at (a) $500 \mathrm{~m}$ and (b) $1200 \mathrm{~m}$ height in the $10 \mathrm{mAR} 1 \mathrm{run}$ of the control experiment.

In all the experiments, the magnitude of the SEP tends to be smaller for the larger-vertical resolution. This tendency is more apparent for the larger aspect ratio. It is possible that the amount of energy dissipation depends on the grid configuration, although it should be identical. The larger dissipation could result in smaller total energy and consequently a smaller SEP in the coarser resolution runs.

\subsection{Turbulence statistics}

In this subsection, we show the influence of the grid configuration of the runs on the turbulence statistics in the PBL in the control experiment. Figure 5 shows the dependency of the vertical profiles of several turbulence statistics on the spatial resolution and grid aspect ratio in the control experiment: the horizontal mean potential temperature, the vertical heat flux, the variance of the vertical velocity, and the skewness of the vertical velocity. These values are averaged over the last half hour $(t=3.5-4 \mathrm{~h})$. The change in the $30 \mathrm{~min}$ averaged kinetic energy at $500 \mathrm{~m}$ in $t=3-3.5 \mathrm{~h}$ to that in $t=3.5-4 \mathrm{~h}$ is small enough: only $3.4 \%$. Consequently, we assume that the state in the last half hour is appropriate enough for the analysis. In the calculation of these values, the horizontal mean is defined as the mass-weighted average, $\frac{\overline{\rho \phi}}{\bar{\rho}}$, where the overline represents the mathematical horizontal average. These
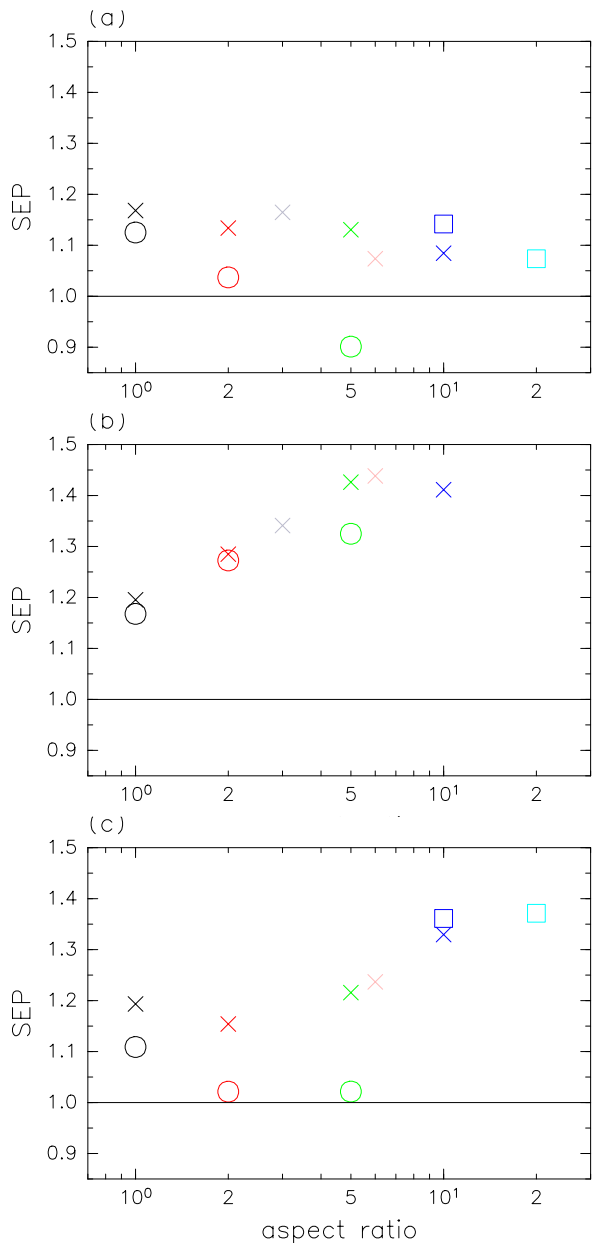

Figure 4. Dependency of the index of energy pile defined in the text on the grid aspect ratio for runs in the (a) control experiment, (b) small filter length experiment, and (c) fixed mixing length experiment. Symbol shows vertical resolution; square, cross, and circle shows 5, 10, and $30 \mathrm{~m}$, respectively. Colors represent the grid aspect ratio as in Fig. 1.

profiles are calculated from the resolved variability, except for the heat flux.

Figure 5a gives the vertical profile of the potential temperature for all the runs. The potential temperature is almost uniform, and the atmosphere is well mixed in the PBL below $1.2 \mathrm{~km}$ height; the difference is about $0.05 \mathrm{~K}$. On the other hand, the profile in the surface layer indicates some difference according to the resolution and grid aspect ratio. Here, we define the surface layer as the layer that keeps the potential temperature gradient less than $-10^{-3} \mathrm{~K} \mathrm{~m}^{-1}$. The conclusions in this paragraph do not change qualitatively for a range of the threshold. In the 10mAR1 run, this steep potential temperature gradient is reproduced in the unstable surface layer below $75 \mathrm{~m}$ depth. Figure 6a shows the dependency of the depth of the surface layer on the horizontal resolution. We found that the depth becomes larger for coarser 

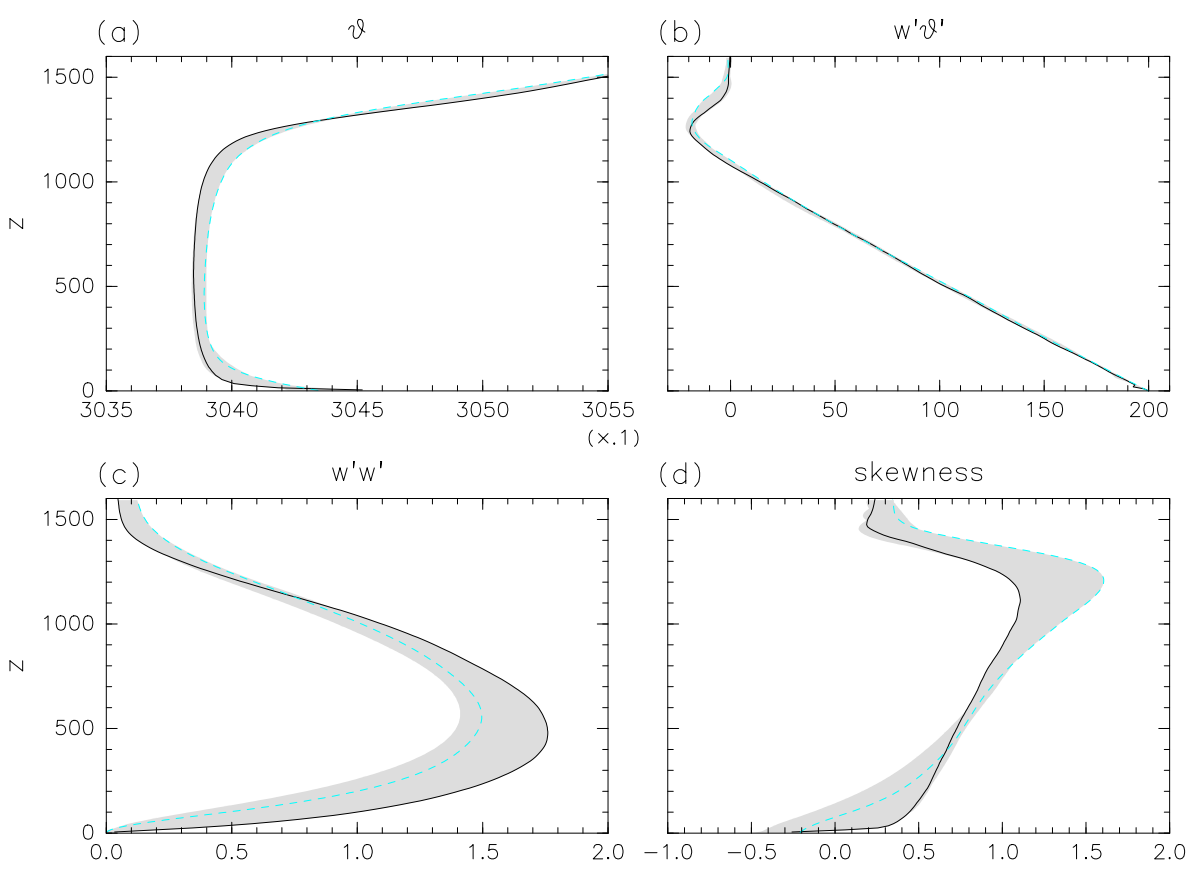

Figure 5. Vertical plots of horizontal mean (a) potential temperature $[\mathrm{K}]$, (b) heat flux $\left[\mathrm{W} \mathrm{m}^{-2}\right]$, (c) variance of vertical velocity $\left[\mathrm{m}^{2} \mathrm{~s}^{-2}\right]$, and (d) skewness of vertical velocity in the control experiment. These values are averaged over $t=3.5$ to $4 \mathrm{~h}$. Black and cyan lines are results of 10mAR1 and 5mAR20 runs, respectively. Shaded areas represent range between maximum and minimum of all runs in the control experiment.

horizontal resolution, even if the vertical resolution is higher. Typically, in the 5mAR20 run, the depth of the surface layer is about $140 \mathrm{~m}$. The variance of the resolved variability is smaller in the 5mAR20 run than in the 10mAR1 run, as shown in Fig. 7, and the strength of mixing by the resolved motion is not a cause of this tendency. Figure 7 shows that the potential temperature and the variance of the horizontal velocity have similar vertical profiles against $z=z / \Delta$ in terms of the vertical gradient. This suggests that the vertical gradient of the profile in the surface layer is determined by the filter length.

Figure $5 \mathrm{~b}$ shows the profile of the vertical heat flux, which is here defined as the sum of the resolved flux, $\overline{\rho w^{\prime} \theta^{\prime}} / \bar{\rho}$, and the SGS flux. Note that the prime represents deviation from the horizontal mean. We define the top boundary of the PBL as the height of minimum heat flux. In all the runs, the PBL height of $1.25 \mathrm{~km}$ and the heat flux profile are almost the same. The flux profile indicates a linear distribution in the $z$ direction, and this means that the heating rate is constant in the PBL. Its slope is about $0.18 \mathrm{~W} \mathrm{~m}^{-3}$, corresponding to a heating rate of about $0.6 \mathrm{Kh}^{-1}$. This value is consistent with the sensible heat flux from the bottom boundary $\left(200 \mathrm{~W} \mathrm{~m}^{-2}\right)$ divided by the depth of the region where the heat flux is positive (about $1.1 \mathrm{~km}$ ). We can conclude that the total vertical heat flux is reasonably reproduced regardless of grid configuration.

The variance of the vertical velocity is shown in Fig. 5c. The variance is largest around $500 \mathrm{~m}$ height in all the runs.
It is 1.75 and $1.4 \mathrm{~m}^{2} \mathrm{~s}^{-2}$ at a height of $500 \mathrm{~m}$ in the highestand lowest-resolution runs (10mAR1 and 30mAR5), respectively. Figure $6 \mathrm{~b}$ shows how the grid configuration affects the variance of vertical velocity. It is found that the variance of the vertical velocity mainly depends on the horizontal resolution; as the horizontal grid spacing decreases, the variance of the vertical velocity increases, and their correlation is about -0.98 . On the other hand, significant dependency of the variance of the vertical velocity on the vertical resolution and grid aspect ratio is not seen; their correlations are -0.21 and -0.56 , respectively.

Theoretically, the variance of vertical velocity should converge to a certain value with grid refinement for the following reason. The variance is approximately equal to the sum of squares of each wave number component as

$\operatorname{Var}_{w} \approx \sum_{k=1}^{k_{\max }}|\hat{w}(k)|^{2}$

where $k_{\max }$ and $\hat{w}(k)$ are the maximum horizontal wave number and amplitude of the vertical velocity of wave number $k$, respectively. Note that here we ignore the density variability for simplicity. Under the condition that the energy spectrum $|\hat{w}|^{2}$ has an exponential decay of $k^{-5 / 3}$, the accumulated energy, which is equal to half of the variance, can be obtained analytically as 

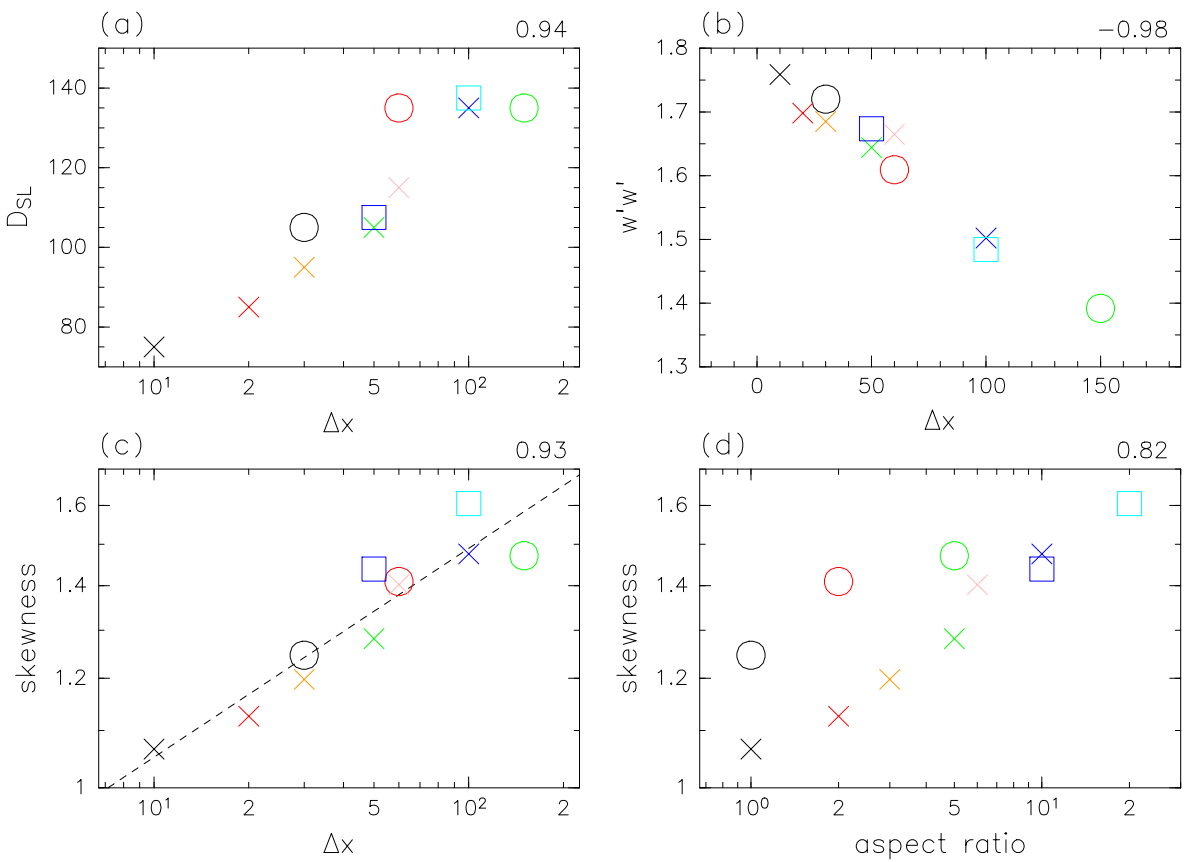

Figure 6. Scatter diagrams between some quantities and grid configurations: (a) depth of surface layer vs. horizontal grid spacing, (b) variance of vertical velocity at $500 \mathrm{~m}$ height vs. horizontal grid spacing, (c) skewness of vertical velocity at $1200 \mathrm{~m}$ height vs. horizontal grid spacing, and (d) skewness vs. grid aspect ratio in the control experiment. Colors and symbols represent grid aspect ratio and vertical resolution, respectively, as in Fig. 4: 1 (black), 2 (red), 3 (orange), 5 (green), 6 (pink), 10 (blue), and 20 (cyan). Squares, crosses, and circles indicate 5, 10, and $30 \mathrm{~m}$, respectively. Value at top right of each diagram is the correlation coefficient. Note that the coefficient is calculated with the logarithm of skewness, horizontal grid spacing, and aspect ratio in (c, d). The broken line in (c) represents the regression line.

$$
\int_{k_{0}}^{k_{\max }} A k^{-5 / 3} \mathrm{~d} k=B-\frac{3}{2} A k_{\max }^{-2 / 3},
$$

where $A$ and $B$ are constants. Nevertheless, as shown in Fig. $6 b$, the variance has not yet converged in the range of resolutions. The convergence point is still a debatable issue at higher resolutions. Here, we try to estimate the convergence point. We estimate the total energy for $k_{\max }=\infty$ from Eq. (24) in the 10mAR1 run, and it is $104 \%$ of the observed total energy in the simulation. This suggests that the convergence point of the variance would be about $1.82 \mathrm{~m}^{2} \mathrm{~s}^{-2}$, i.e., $104 \%$ of $1.75 \mathrm{~m}^{2} \mathrm{~s}^{-2}$.

The profile of skewness shows an almost linear slope in the PBL except near the surface, as shown in Fig. 5d. The skewness has a similar value around $500 \mathrm{~m}$ height for all the runs, while it shows variety around the top and bottom of the PBL. Positive skewness implies stronger upward motion compared to downward motion. Around the top boundary, the hexagonal or quadrangular cell structure of convection seen around the middle of the layer in Fig. $3 \mathrm{a}$ is no longer dominant, as Fig. $3 \mathrm{~b}$ shows. There are strong upward plumes surrounded by compensating annular downward flow, whose horizontal scale is about 1-2 km. Most of the plumes are located above the cell vertex at lower height. Figure $6 \mathrm{c}$ shows the dependency of the skewness on the horizontal resolution at $1.2 \mathrm{~km}$ height (top of the PBL). The skewness is larger for coarser resolution. The logarithmic values of skewness and horizontal resolution have a mostly linear relationship. Sullivan and Patton (2011) tried to explain the dependency of the skewness on the horizontal resolution by the SGS moment, but they showed that its effect is quantitatively not sufficient to explain the difference. We suppose that localized strong upward plumes due to the larger eddy viscosity is a possible cause of the larger skewness based on the following explanation. Coarser resolution corresponds to larger filter length and consequently, larger eddy viscosity. The larger viscosity prevents small-scale motions. However, the amount of heat transferred vertically should be almost the same as in other runs, since static instability becomes strong if the vertical heat flux is smaller because motion is prevented. In fact, the horizontal mean vertical heat flux is almost identical in all the runs (Fig. 5b). Individual convective plumes in coarser resolution runs could be stronger, transferring more heat than in higher resolution runs. It is possible that such localized stronger upward plumes are the cause of the larger skewness in coarser resolution runs.

Residuals from logarithmic linear regression of the skewness in the horizontal resolution are relatively larger for coarser horizontal resolution: e.g., the 5mAR20 (cyan square) and the 30mAR5 (green circle) runs. Dependency 

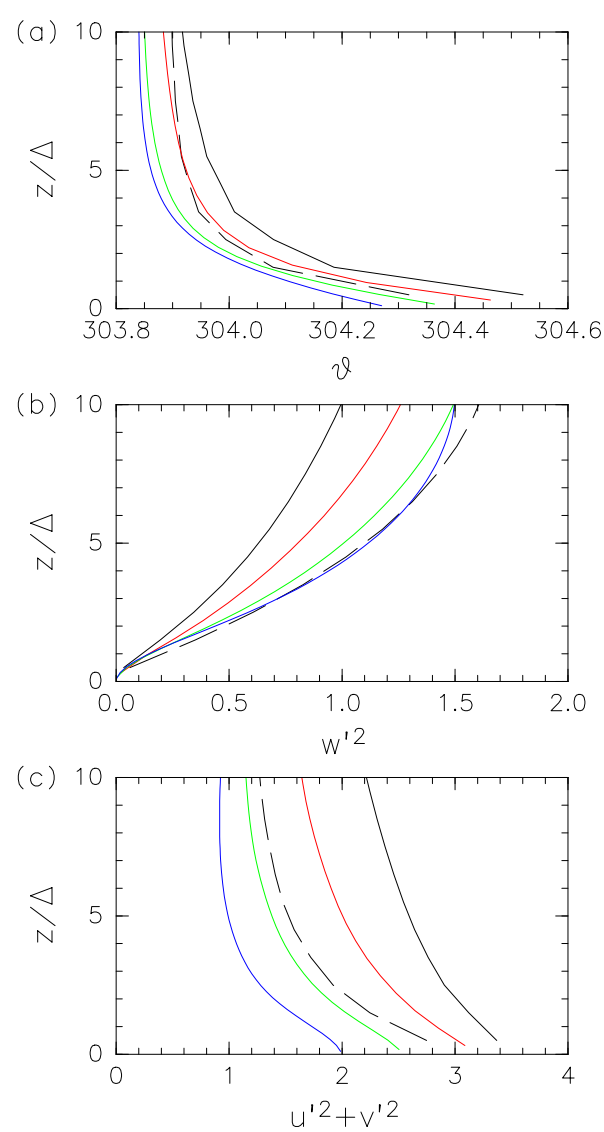

Figure 7. The vertical profiles against $z / \Delta$ of (a) the potential temperature, (b) variance of the vertical velocity, and (c) variance of the horizontal velocity in the 10mAR1 (black solid line), 10mAR2 (red solid), 10mAR5 (green solid), 10mAR10 (blue solid), and 30mAR1 (black broken) runs.

of the skewness on the grid aspect ratio is one of the reasons for the residuals. The dependency on the aspect ratio can be seen in Fig. 6d. The skewness tends to be larger for larger aspect ratios. Positive residuals from the 5mAR20 run and negative ones from the 30mAR5 run in the regression would be due to large and small aspect ratios, respectively. Also, the skewness of the 5mAR20 run is larger than that of the 10mAR10 run (blue $\times$ symbol) at the same horizontal resolutions $(100 \mathrm{~m})$. Their difference could be due to the difference in aspect ratio. The skewness in the 10mAR10 run is closer to that of the 10mAR1 run (black $\times$ symbol), than that of the 5mAR20 run, even if the vertical resolution is higher in the 5mAR20 run than in the 10mAR10 run. This suggests that a large aspect ratio produces spuriously large skewness in vertical velocity, that is, artificially strong upwind. The dependence on the grid aspect ratio seems somewhat complex, and the reason for the dependence is not clear. We assume that it is related to static stability. For higher grid aspect ratios, i.e., relatively higher vertical resolution or lower horizontal resolution, static stability could vary verti- cally rather than horizontally. In that case, a strong shallow stable layer could cover a relatively wider region horizontally, and strong static stability and small horizontal contrast might prevent vertical motion and suppress the vertical eddy viscosity and diffusion. Under this condition, only a strong parcel can go upward against the thin strong stable layer. For the same amount of vertical heat transportation independent of grid configuration, a larger grid aspect ratio might require stronger upwind, indicating larger skewness.

\section{Conclusions}

We conducted a series of planetary boundary layer experiments to examine the influences of the aspect ratio of horizontal to vertical grid spacing on the atmospheric turbulence in a large-eddy simulation. In order to focus on the influences, we tried to avoid artificial effects as much as possible by employing the fully compressible governing equations. A fully explicit (i.e., HE-VE) temporal integration scheme and a high-order central difference scheme for spatial differentials are adopted to reduce implicitly introduced numerical viscosity and diffusion by discretization.

In the model used in this paper, we considered the effect of spatial filter length and grid aspect ratio on the mixing length of the eddy viscosity and diffusion, which is a parameterization of the energy cascade to SGS variability. Explicit numerical hyperviscosity and diffusion is introduced to reduce the two-grid-scale noise in this model. This can be considered a spatial filter in LES. As a result, the filter length in this model is double the grid spacing, while the grid spacing is used as the length in most LESs. The effect of the grid aspect ratio on the mixing length proposed by Scotti et al. (1993) was also introduced.

If we use a reasonable filter length and introduce the grid aspect ratio effect, the energy spectra of all the runs show good correspondence with the theoretical $k^{-5 / 3}$ slope, regardless of spatial resolution and grid aspect ratio. On the other hand, the theoretical slope in the spectrum is no longer obtained, and a spurious energy pile is found if the spatial filter length is set to be equal to the grid spacing as in most meteorological LESs. The spurious energy piling is more significant for larger grid aspect ratios. A shorter filter length results in a shorter mixing length and consequently, a smaller energy cascade to SGS variability. Thus, an inappropriate filter length causes spurious energy.

The effect of the grid aspect ratio on the mixing length of the eddy viscosity and diffusion cannot be ignored. If the mixing length is not modified by the grid aspect ratio, spurious energy also piles at higher wave numbers because of an insufficient energy cascade to SGS turbulence at large grid aspect ratios. In previous studies, the Smagorinsky constant was often modified as a tuning parameter to obtain the expected energy spectrum. However, the constant should be determined theoretically and should not be tuned to make the 
energy cascade large instead of considering filter length and grid aspect ratio.

The horizontal resolution and grid aspect ratio also influence the turbulence statistics. The vertical profiles of several turbulence statistics depend on the grid configuration as follows. The depth of the unstable surface layer is larger for coarser horizontal resolution (but not vertical resolution). The variance of the resolved vertical velocity essentially depends on the horizontal resolution, mainly because of spatial averaging by discretization. The skewness of the vertical velocity shows dependency on grid aspect ratio as well as on horizontal resolution. In particular, the skewness is sensitive to the grid configuration around the top and bottom of the PBL. It becomes larger and smaller around the top and bottom, respectively, for coarser resolution and larger grid aspect ratio. Higher resolution and smaller grid aspect ratio are required in order to obtain accurate skewness. This is im- portant, because the spurious strong upward wind near the PBL top, which is implied by larger skewness, would have a large effect on cloud microphysical processes. For example, the reproducibility of clouds around the PBL top, such as stratocumulus, would be sensitive to grid configuration.

We conclude that the grid aspect ratio influences the parameterization of the energy cascade to SGS variability and the reproducibility of skewness of turbulence in the PBL. Although there are many meteorological LESs in which the grid aspect ratio is large, such large grid aspect ratios have led to misinterpretation in some experiments in previous studies because of spurious energy piling at higher wave numbers and stronger vertical motion indicated by larger skewness. The aspect ratio should be taken into account properly in determining the mixing length of the eddy viscosity and diffusion as a sub-grid model for the reliability of simulations of boundary layer turbulence. 


\section{Appendix A: Model description}

\section{A1 Governing equations}

In this subsection, we introduce the governing equations for the prognostic variables $\left(\rho, \rho u, \rho v, \rho w, \rho \theta\right.$, and $\left.\rho q_{x} \mathrm{~s}\right)$. The gas constant and specific heat are those for total (moist) air in the thermodynamics equation and equation of state. The $\theta$ in this model is not the conventional potential temperature for dry air, but the corresponding value for total air, considering water content. These variables are spatially filtered quantities, and the Favre filter (Favre, 1983) is used for $u, v, w, \theta$, and $q_{x} \mathrm{~s}$.

\section{A1.1 Continuity equations}

The continuity equations for each material can be described in flux form:

$$
\begin{aligned}
& \frac{\partial \rho q_{\mathrm{d}}}{\partial t}+\nabla \cdot\left(\rho q_{\mathrm{d}} \boldsymbol{u}\right)=\operatorname{DIFF}\left[q_{\mathrm{d}}\right], \\
& \frac{\partial \rho q_{\mathrm{v}}}{\partial t}+\nabla \cdot\left(\rho q_{\mathrm{v}} \boldsymbol{u}\right)=S_{\mathrm{v}}+\operatorname{DIFF}\left[q_{\mathrm{v}}\right], \\
& \frac{\partial \rho q_{1}}{\partial t}+\nabla \cdot\left(\rho q_{\mathrm{l}} \boldsymbol{u}\right)+\frac{\partial \rho q_{\mathrm{l}} w_{1}}{\partial z}=S_{1}+\operatorname{DIFF}\left[q_{1}\right], \\
& \frac{\partial \rho q_{\mathrm{s}}}{\partial t}+\nabla \cdot\left(\rho q_{\mathrm{s}} \boldsymbol{u}\right)+\frac{\partial \rho q_{\mathrm{s}} w_{\mathrm{s}}}{\partial z}=S_{\mathrm{s}}+\operatorname{DIFF}\left[q_{\mathrm{s}}\right],
\end{aligned}
$$

where $\nabla$ is the gradient vector, and DIFF $[\phi]$ is the diffusion term by SGS turbulence (see Sect. 2.2). The sum of the ratios of their mass should be unity:

$q_{\mathrm{d}}+q_{\mathrm{v}}+q_{1}+q_{\mathrm{s}}=1$.

The source terms for water substances should satisfy the following relationship:

$S_{\mathrm{v}}+S_{1}+S_{\mathrm{s}}=0$.

The sum of Eqs. (A1)-(A4) gives the continuity equation of total density:

$$
\frac{\partial \rho}{\partial t}+\nabla \cdot(\rho \boldsymbol{u})+\frac{\partial \rho q_{1} w_{1}}{\partial z}+\frac{\partial \rho q_{\mathrm{s}} w_{\mathrm{s}}}{\partial z}=0 .
$$

For this derivation, we use the fact that the operator DIFF $[\phi]$ is a linear operator:

$$
\operatorname{DIFF}[\phi]=-\frac{\partial}{\partial x_{i}} \rho v_{\mathrm{SGS}}^{*} \frac{\partial \phi}{\partial x_{i}} .
$$

Using Eq. (A5),

$$
\begin{gathered}
\operatorname{DIFF}\left[q_{\mathrm{d}}\right]+\operatorname{DIFF}\left[q_{\mathrm{v}}\right]+\operatorname{DIFF}\left[q_{1}\right]+\operatorname{DIFF}\left[q_{\mathrm{s}}\right] \\
\quad=\operatorname{DIFF}\left[q_{\mathrm{d}}+q_{\mathrm{v}}+q_{1}+q_{\mathrm{s}}\right]=\operatorname{DIFF}[1]=0 .
\end{gathered}
$$

\section{A1.2 Momentum equations}

The momentum equations for the gas, liquid, and solid materials are described as

$$
\begin{aligned}
& \frac{\partial \rho\left(q_{\mathrm{d}}+q_{\mathrm{v}}\right) \boldsymbol{u}}{\partial t}+\nabla \cdot\left[\rho\left(q_{\mathrm{d}}+q_{\mathrm{v}}\right) \boldsymbol{u} \otimes \boldsymbol{u}\right] \\
& \quad=-\nabla p-\left[\rho\left(q_{\mathrm{d}}+q_{\mathrm{v}}\right) g+\left(f_{\mathrm{l}}+f_{\mathrm{s}}\right)\right] \boldsymbol{e}_{z}+\boldsymbol{u} S_{\mathrm{v}} \\
& \quad+\operatorname{DIFF}\left[\left(q_{\mathrm{d}}+q_{\mathrm{v}}\right) \boldsymbol{u}\right], \\
& \frac{\partial \rho q_{\mathrm{l}} \boldsymbol{u}}{\partial t}+\nabla \cdot\left(\rho q_{\mathrm{l}} \boldsymbol{u} \otimes \boldsymbol{u}\right)+\frac{\partial \rho q_{\mathrm{l}} \boldsymbol{u} w_{\mathrm{l}}}{\partial z} \\
& \quad=-\left(\rho q_{1} g-f_{\mathrm{l}}\right) \boldsymbol{e}_{z}+\boldsymbol{u} S_{\mathrm{l}}+\operatorname{DIFF}\left[q_{\mathrm{l}} \boldsymbol{u}\right], \\
& \frac{\partial \rho q_{\mathrm{s}} \boldsymbol{u}}{\partial t}+\nabla \cdot\left(\rho q_{\mathrm{s}} \boldsymbol{u} \otimes \boldsymbol{u}\right)+\frac{\partial \rho q_{\mathrm{s}} \boldsymbol{u} w_{\mathrm{s}}}{\partial z} \\
& \quad=-\left(\rho q_{\mathrm{s}} g-f_{\mathrm{s}}\right) \boldsymbol{e}_{z}+\boldsymbol{u} S_{\mathrm{s}}+\operatorname{DIFF}\left[q_{\mathrm{s}} \boldsymbol{u}\right],
\end{aligned}
$$

where $\otimes$ represents the tensor product. The pressure is derived from the equation of state as

$p=\rho\left(q_{\mathrm{d}} R_{\mathrm{d}}+q_{\mathrm{v}} R_{\mathrm{V}}\right) T$.

The sum of Eqs. (A10)-(A12) gives the total momentum equation as

$$
\begin{aligned}
\frac{\partial \rho \boldsymbol{u}}{\partial t} & +\nabla \cdot(\rho \boldsymbol{u} \otimes \boldsymbol{u})+\left(\frac{\partial \rho q_{1} w_{1}}{\partial z}+\frac{\partial \rho q_{\mathrm{s}} w_{\mathrm{s}}}{\partial z}\right) \boldsymbol{u} \\
= & -\nabla p-\rho g \boldsymbol{e}_{z}+\operatorname{DIFF}[\boldsymbol{u}] .
\end{aligned}
$$

Note that the drag forces from loading do not appear in Eq. (A14), because those terms are canceled out by the summation.

\section{A1.3 Thermodynamics equations}

The equations of internal energies are given as

$$
\begin{aligned}
& \frac{\partial \rho\left(q_{\mathrm{d}} e_{\mathrm{d}}+q_{\mathrm{v}} e_{\mathrm{v}}\right)}{\partial t}+\nabla \cdot\left[\rho\left(q_{\mathrm{d}} e_{\mathrm{d}}+q_{\mathrm{v}} e_{\mathrm{v}}\right) \boldsymbol{u}\right] \\
& =-p \nabla \cdot \boldsymbol{u}+Q_{d}+Q_{v}+\operatorname{DIFF}\left[\left(q_{\mathrm{d}}+q_{\mathrm{v}}\right) T^{*}\right], \\
& \frac{\partial \rho q_{1} e_{1}}{\partial t}+\nabla \cdot\left(\rho q_{1} e_{1} \boldsymbol{u}\right)+\frac{\partial \rho q_{1} e_{1} w_{1}}{\partial z} \\
& \quad=Q_{1}+\operatorname{DIFF}\left[q_{1} T^{*}\right], \\
& \frac{\partial \rho q_{1} e_{\mathrm{s}}}{\partial t}+\nabla \cdot\left(\rho q_{\mathrm{s}} e_{\mathrm{s}} \boldsymbol{u}\right)+\frac{\partial \rho q_{\mathrm{s}} e_{\mathrm{s}} w_{\mathrm{s}}}{\partial z} \\
& \quad=Q_{\mathrm{s}}+\operatorname{DIFF}\left[q_{\mathrm{s}} T^{*}\right],
\end{aligned}
$$

where $e_{x}$ are the internal energies, and defined as

$$
\begin{aligned}
& e_{\mathrm{d}}=c_{v \mathrm{~d}} T, \\
& e_{\mathrm{v}}=c_{v \mathrm{v}} T, \\
& e_{1}=c_{1} T, \\
& e_{\mathrm{S}}=c_{\mathrm{s}} T .
\end{aligned}
$$

The DIFF operator represents the mixing by SGS turbulence. The SGS eddy viscosity should be used for conserved quantities following the motion. The internal energies are not conserved quantities in the Lagrangian sense, and the diffusion 
term in these equations and $T^{*}$ are only conceptual, and should be determined to be consistent with the diffusion term in an equation for a conserved quantity along the flow trajectory (i.e., potential temperature), which is discussed later.

The sum of Eqs. (A15)-(A17) gives the total internal energy equations

$$
\begin{aligned}
& \frac{\partial \rho e}{\partial t}+\nabla \cdot(\rho e \boldsymbol{u})+\frac{\partial \rho q_{1} e_{1} w_{1}}{\partial z}+\frac{\partial \rho q_{\mathrm{s}} e_{\mathrm{s}} w_{\mathrm{s}}}{\partial z} \\
& +p \boldsymbol{\nabla} \cdot \boldsymbol{u}=Q+\operatorname{DIFF}\left[T^{*}\right],
\end{aligned}
$$

where

$$
e=q_{\mathrm{d}} e_{\mathrm{d}}+q_{\mathrm{v}} e_{\mathrm{v}}+q_{1} e_{1}+q_{\mathrm{s}} e_{\mathrm{s}},
$$

and the total diabatic heating is described as

$$
Q=Q_{\mathrm{d}}+Q_{\mathrm{v}}+Q_{1}+Q_{\mathrm{s}}
$$

Equations (A2)-(A4), (A7), (A14), and (A22), along with Eq. (A13), form the complete set of equations.

\section{A1.4 Conservation of thermodynamics in the dynamical process}

Equation (A22) is not a complete flux form, because the internal energy itself is not conserved both in the Euler sense and the Lagrangian sense. In this section, we consider the conserved quantity for the thermodynamics equation.

The potential temperature for dry air, which is defined as

$\theta_{\mathrm{d}}=T\left(\frac{p_{00}}{p}\right)^{R_{\mathrm{d}} / c_{p \mathrm{~d}}}$

is used as a conserved quantity in traditional models. Although it is conserved along a Lagrangian trajectory, it is no longer conserved when the water substances are included. We introduce a new conserved quantity following the motion in moist conditions.

Using Eqs. (A7) and (A22), without the terms for the sedimentation of water, the diabatic heating and diffusion can be written as

$$
\frac{\mathrm{d} e}{\mathrm{~d} t}+p \frac{\mathrm{d}}{\mathrm{d} t}\left(\frac{1}{\rho}\right)=0
$$

Substituting Eqs. (A13), (A18)-(A21), and (A23) into Eq. (A26),

$$
\begin{aligned}
& \frac{\mathrm{d} q_{\mathrm{d}} c_{v \mathrm{~d}} T}{\mathrm{~d} t}+p \frac{\mathrm{d}}{\mathrm{d} t}\left[\frac{q_{\mathrm{d}} R_{\mathrm{d}} T}{p}\right]+\frac{\mathrm{d} q_{\mathrm{v}} c_{v \mathrm{v}} T}{\mathrm{~d} t}+p \frac{\mathrm{d}}{\mathrm{d} t}\left[\frac{q_{\mathrm{v}} R_{\mathrm{v}} T}{p}\right] \\
& +\frac{\mathrm{d} q_{1} c_{1} T}{\mathrm{~d} t}+\frac{\mathrm{d} q_{\mathrm{s}} c_{\mathrm{s}} T}{\mathrm{~d} t}=0 .
\end{aligned}
$$

From Eqs. (A2)-(A4) and (A7), Eq. (A27) gives the following form:

$q_{\mathrm{d}}\left[\frac{\mathrm{d} c_{v \mathrm{~d}} T}{\mathrm{~d} t}+p \frac{\mathrm{d}}{\mathrm{d} t}\left[\frac{R_{\mathrm{d}} T}{p}\right]\right]+q_{\mathrm{v}}\left[\frac{\mathrm{d} c_{v \mathrm{v}} T}{\mathrm{~d} t}+p \frac{\mathrm{d}}{\mathrm{d} t}\left[\frac{R_{\mathrm{v}} T}{p}\right]\right]$

$$
+q_{1} \frac{\mathrm{d} c_{1} T}{\mathrm{~d} t}+q_{\mathrm{s}} \frac{\mathrm{d} c_{\mathrm{s}} T}{\mathrm{~d} t}=0 .
$$

Dividing this equation by $T$,

$$
\begin{gathered}
q_{\mathrm{d}} c_{p \mathrm{~d}}\left[\frac{\mathrm{d} \ln T}{\mathrm{~d} t}+\frac{R_{\mathrm{d}}}{c_{p \mathrm{~d}}} \frac{\mathrm{d}}{\mathrm{d} t}\left[\ln \left(\frac{1}{p}\right)\right]\right] \\
+q_{\mathrm{v}} c_{p \mathrm{v}}\left[\frac{\mathrm{d} \ln T}{\mathrm{~d} t}+\frac{R_{\mathrm{v}}}{c_{p \mathrm{v}}} \frac{\mathrm{d}}{\mathrm{d} t}\left[\ln \left(\frac{1}{p}\right)\right]\right] \\
+q_{1} c_{1} \frac{\mathrm{d} \ln T}{\mathrm{~d} t}+q_{\mathrm{s}} c_{\mathrm{s}} \frac{\mathrm{d} \ln T}{\mathrm{~d} t}=0,
\end{gathered}
$$

and

$$
\begin{aligned}
& q_{\mathrm{d}} c_{p \mathrm{~d}} \frac{\mathrm{d} \ln \theta_{\mathrm{d}}}{\mathrm{d} t}+q_{\mathrm{v}} c_{p \mathrm{v}} \frac{\mathrm{d} \ln \theta_{\mathrm{v}}}{\mathrm{d} t}+q_{1} c_{1} \frac{\mathrm{d} \ln T}{\mathrm{~d} t} \\
& +q_{\mathrm{s}} c_{\mathrm{s}} \frac{\mathrm{d} \ln T}{\mathrm{~d} t}=0,
\end{aligned}
$$

where $\theta_{\mathrm{v}}$ is the potential temperature for water vapor, defined as

$\theta_{\mathrm{v}}=T\left(\frac{p_{00}}{p}\right)^{R_{\mathrm{v}} / c_{p \mathrm{v}}}$.

Thus,

$\frac{\mathrm{d}}{\mathrm{d} t}\left[\ln \left(\theta_{\mathrm{d}}^{q_{\mathrm{d}} c_{p \mathrm{~d}}} \theta_{\mathrm{v}}^{q_{\mathrm{v}} c_{p \mathrm{v}}} T^{q_{\mathrm{l}} c_{1}} T^{q_{\mathrm{s}} c_{\mathrm{s}}}\right)\right]=0$.

The quantity

$\Theta=\theta_{\mathrm{d}}^{q_{\mathrm{d}} c_{p \mathrm{~d}}} \theta_{\mathrm{v}}^{q_{\mathrm{v}} c_{p \mathrm{v}}} T^{q_{1} c_{1}} T^{q_{\mathrm{s}} c_{\mathrm{s}}}=T^{c_{p}^{*}}\left(\frac{p_{00}}{p}\right)^{R^{*}}$

is conserved along the flow trajectory, where

$c_{p}^{*} \equiv q_{\mathrm{d}} c_{p \mathrm{~d}}+q_{\mathrm{v}} c_{p \mathrm{v}}+q_{1} c_{1}+q_{\mathrm{s}} c_{\mathrm{s}}$,

$R^{*} \equiv q_{\mathrm{d}} R_{\mathrm{d}}+q_{\mathrm{v}} R_{\mathrm{v}}$.

Here we define a new potential temperature

$\theta \equiv \Theta^{1 / c_{p}^{*}}=T\left(\frac{p_{00}}{p}\right)^{R^{*} / c_{p}^{*}}$.

This $\theta$ satisfies

$\frac{\mathrm{d} \theta}{\mathrm{d} t}=\frac{1}{c_{p}^{*}} \Theta^{1 / c_{p}^{*}-1} \frac{\mathrm{d} \Theta}{\mathrm{d} t}=0$,

and $\theta$ is a conserved quantity along the flow trajectory, even in moist conditions. We employ $\rho \theta$ for the prognostic variable.

The pressure expression is derived diagnostically as

$p=p_{00}\left(\frac{\rho \theta R^{*}}{p_{00}}\right)^{\frac{c_{p}^{*}}{c_{p}^{*}-R^{*}}}$.

Figure A1a gives the vertical profile of the temperature in the US control atmosphere, and Fig. A1b shows the vertical profiles of $\theta / \theta_{\mathrm{d}}$ under this temperature condition when we assume that $q_{\mathrm{v}}$ is the specific humidity at saturation, and $q_{1}+$ $q_{\mathrm{s}}$ gives $0.0,0.01,0.02$, and 0.04 . The difference between $\theta$ and $\theta_{\mathrm{d}}$ becomes larger with height and may not be negligible. 

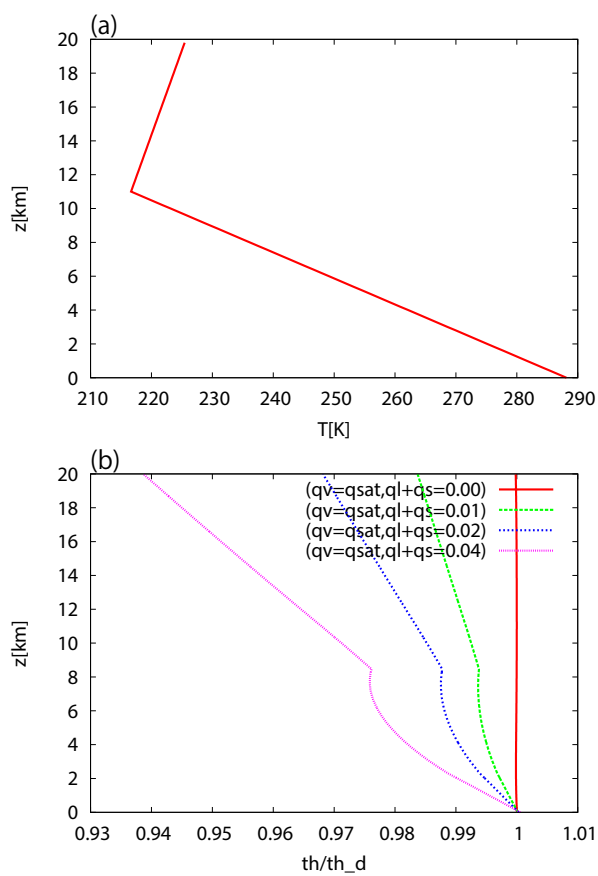

Figure A1. Vertical profile of (a) US standard atmosphere; (b) several profiles of $\theta / \theta_{\mathrm{d}}$.

\section{A1.5 Diabatic heating in the physical process}

Changing the prognostic variable for thermodynamics from the internal energy to the newly defined potential temperature $\theta$ should modify the diabatic heating and sedimentation terms in Eq. (A22). Through the manipulation of Eqs. (A26) to (A32), Eq. (A22) can be written as

$$
\begin{aligned}
& \rho T \frac{\mathrm{d} \ln \Theta}{\mathrm{d} t}=Q-\frac{\partial \rho q_{1} e_{1} w_{1}}{\partial z}-\frac{\partial \rho q_{\mathrm{s}} e_{\mathrm{s}} w_{\mathrm{s}}}{\partial z} \\
& \quad+\operatorname{DIFF}\left[T^{*}\right] .
\end{aligned}
$$

Substituting Eq. (A36) into Eq. (A39),

$$
\begin{aligned}
& \rho \frac{\mathrm{d} \theta}{\mathrm{d} t}=\frac{1}{c_{p}^{*}}\left(\frac{p_{00}}{p}\right)^{\frac{R^{*}}{c_{p}^{*}}} \\
& \left(Q-\frac{\partial \rho q_{1} e_{1} w_{1}}{\partial z}-\frac{\partial \rho q_{\mathrm{s}} e_{\mathrm{s}} w_{\mathrm{s}}}{\partial z}+\operatorname{DIFF}\left[T^{*}\right]\right) .
\end{aligned}
$$

$T^{*}$ is the variable defined to satisfy the following equation

$$
\frac{1}{c_{p}^{*}}\left(\frac{p_{00}}{p}\right)^{\frac{R^{*}}{c_{p}^{*}}} \operatorname{DIFF}\left[T^{*}\right]=\operatorname{DIFF}[\theta] .
$$

\section{A1.6 Summary of governing equations}

The governing equations are summarized as follows:

$$
\frac{\partial \rho q_{\mathrm{v}}}{\partial t}=-\nabla \cdot\left(\rho q_{\mathrm{v}} \boldsymbol{u}\right)+S_{\mathrm{v}}+\operatorname{DIFF}\left[q_{\mathrm{v}}\right],
$$

$$
\begin{aligned}
\frac{\partial \rho q_{1}}{\partial t}= & -\nabla \cdot\left(\rho q_{1} \boldsymbol{u}\right)-\frac{\partial \rho q_{1} w_{1}}{\partial z}+S_{1}+\operatorname{DIFF}\left[q_{1}\right], \\
\frac{\partial \rho q_{\mathrm{s}}}{\partial t}= & -\nabla \cdot\left(\rho q_{\mathrm{s}} \boldsymbol{u}\right)-\frac{\partial \rho q_{\mathrm{s}} w_{\mathrm{s}}}{\partial z}+S_{\mathrm{s}}+\operatorname{DIFF}\left[q_{\mathrm{s}}\right], \\
\frac{\partial \rho}{\partial t}= & -\nabla \cdot(\rho \boldsymbol{u})-\frac{\partial \rho q_{1} w_{1}}{\partial z}-\frac{\partial \rho q_{\mathrm{s}} w_{\mathrm{s}}}{\partial z}, \\
\frac{\partial \rho \boldsymbol{u}}{\partial t}= & -\nabla \cdot(\rho \boldsymbol{u} \otimes \boldsymbol{u})-\nabla p-\rho g \boldsymbol{e}_{z}-\frac{\partial \rho q_{1} \boldsymbol{u} w_{1}}{\partial z} \\
& -\frac{\partial \rho q_{\mathrm{s}} \boldsymbol{u} w_{\mathrm{s}}}{\partial z}+\operatorname{DIFF}[\boldsymbol{u}], \\
\frac{\partial \rho \theta}{\partial t}= & -\nabla \cdot(\rho \theta \boldsymbol{u}) \\
& +\frac{1}{c_{p}^{*}}\left(\frac{p_{00}}{p}\right)^{\frac{R^{*}}{c_{p}^{*}}}\left[Q-\frac{\partial \rho q_{1} e_{1} w_{1}}{\partial z}-\frac{\partial \rho q_{\mathrm{s}} e_{\mathrm{s}} w_{\mathrm{s}}}{\partial z}\right] \\
& +\operatorname{DIFF}[\theta],
\end{aligned}
$$

where

$p=p_{00}\left(\frac{\rho \theta R^{*}}{p_{00}}\right)^{\frac{c_{p}^{*}}{c_{p}^{*}-R^{*}}}$,

$c_{p}^{*} \equiv q_{\mathrm{d}} c_{p \mathrm{~d}}+q_{\mathrm{v}} c_{p \mathrm{v}}+q_{1} c_{1}+q_{\mathrm{s}} c_{\mathrm{s}}$,

$R^{*} \equiv q_{\mathrm{d}} R_{\mathrm{d}}+q_{\mathrm{v}} R_{\mathrm{v}}$.

\section{A1.7 Boundary condition}

The vertical boundary conditions are that the vertical velocities at the top and bottom boundaries are zero. This causes the vertical flux at the top and bottom boundaries for all the prognostic variables to be zero:

$w=0$,

$\rho w=0$,

$\rho q_{\mathrm{v}} w=\rho q_{1} w=\rho q_{\mathrm{s}} w=0$,

$\rho u w=\rho v w=\rho w w=0$,

$\rho \theta w=0$,

at the top and bottom boundaries.

\section{A2 Temporal integration scheme}

We conceptually separate the complete set of governing equations into dynamical and physical parts:

$$
\frac{\partial \phi}{\partial t}=\left(\frac{\partial \phi}{\partial t}\right)_{\text {dynamics }}+\left(\frac{\partial \phi}{\partial t}\right)_{\text {physics }} .
$$

The diabatic heating process, diffusion by SGS turbulence, sedimentation process of liquid and solid waters, and the source and sink of water substances are treated as physical processes, and the others are treated as dynamical processes.

According to this scheme, the dynamical processes can be written as

$\frac{\partial \rho q_{\mathrm{v}}}{\partial t}+\nabla \cdot\left(\rho q_{\mathrm{v}} \boldsymbol{u}\right)=0$, 


$$
\begin{aligned}
& \frac{\partial \rho q_{1}}{\partial t}+\nabla \cdot\left(\rho q_{1} \boldsymbol{u}\right)=0, \\
& \frac{\partial \rho q_{\mathrm{s}}}{\partial t}+\nabla \cdot\left(\rho q_{\mathrm{s}} \boldsymbol{u}\right)=0, \\
& \frac{\partial \rho}{\partial t}+\nabla \cdot(\rho \boldsymbol{u})=0, \\
& \frac{\partial \rho \boldsymbol{u}}{\partial t}+\nabla \cdot(\rho \boldsymbol{u} \otimes \boldsymbol{u})=-\nabla p-\rho g \boldsymbol{e}_{z} \\
& \frac{\partial \rho \theta}{\partial t}+\nabla \cdot(\rho \theta \boldsymbol{u})=0 .
\end{aligned}
$$

On the other hand, the physical processes are as follows. The governing equations for the physical processes being

$$
\begin{aligned}
\frac{\partial \rho q_{\mathrm{v}}}{\partial t} & =S_{\mathrm{v}}+\operatorname{DIFF}\left[q_{\mathrm{v}}\right], \\
\frac{\partial \rho q_{1}}{\partial t}= & =-\frac{\partial \rho q_{1} w_{1}}{\partial z}+S_{1}+\operatorname{DIFF}\left[q_{1}\right], \\
\frac{\partial \rho q_{\mathrm{s}}}{\partial t} & =-\frac{\partial \rho q_{\mathrm{s}} w_{\mathrm{s}}}{\partial z}+S_{\mathrm{s}}+\operatorname{DIFF}\left[q_{\mathrm{s}}\right], \\
\frac{\partial \rho}{\partial t}= & -\frac{\partial \rho q_{1} w_{1}}{\partial z}-\frac{\partial \rho q_{\mathrm{s}} w_{\mathrm{s}}}{\partial z}, \\
\frac{\partial \rho \boldsymbol{u}}{\partial t}= & -\frac{\partial \rho q_{1} \boldsymbol{u} w_{1}}{\partial z}-\frac{\partial \rho q_{\mathrm{s}} \boldsymbol{u} w_{\mathrm{s}}}{\partial z}+\operatorname{DIFF}[\boldsymbol{u}], \\
\frac{\partial \rho \theta}{\partial t}= & \frac{1}{c_{p}^{*}}\left(\frac{p_{00}}{p}\right)^{\frac{R^{*}}{c_{p}^{*}}}\left[Q-\frac{\partial \rho q_{1} e_{1} w_{1}}{\partial z}-\frac{\partial \rho q_{\mathrm{s}} e_{\mathrm{s}} w_{\mathrm{s}}}{\partial z}\right] \\
& +\operatorname{DIFF}[\theta] .
\end{aligned}
$$

\section{A2.1 Dynamical processes}

A Runge-Kutta (RK) scheme with three steps is used as the temporal integration scheme for the dynamical processes. The RK scheme with three steps used in this model is defined as

$k_{1}=\Delta t f\left(\phi^{t}\right)$,

$k_{2}=\Delta t f\left(\phi^{t}+\frac{1}{3} k_{1}\right)$,

$k_{3}=\Delta t f\left(\phi^{t}+\frac{1}{2} k_{2}\right)$,

$\phi^{t+\Delta t}=\phi^{t}+k_{3}$,

where $f(\phi) \equiv \frac{\partial \phi}{\partial t}$. Taylor expansion of the $\phi^{t+\Delta t}$ calculated by Eq. (A72) around $\phi^{t}$ yields

$$
\begin{aligned}
\phi^{t+\Delta t}= & \phi^{t}+\Delta t f\left(\phi^{t}\right)+\frac{1}{2} \Delta t^{2} f\left(\phi^{t}\right) f^{\prime}\left(\phi^{t}\right) \\
& +\frac{1}{6} \Delta t^{3} f\left(\phi^{t}\right)\left\{f^{\prime}\left(\phi^{t}\right)\right\}^{2}+\frac{1}{8} \Delta t^{3}\left\{f\left(\phi^{t}\right)\right\}^{2} f^{\prime \prime}\left(\phi^{t}\right) \\
& +O\left(\Delta t^{4}\right) .
\end{aligned}
$$

Theoretically, the Taylor expansion of $\phi(t+\Delta t)$ is

$\phi(t+\Delta t)=\phi^{t}+\Delta t f\left(\phi^{t}\right)+\frac{1}{2} \Delta t^{2} f\left(\phi^{t}\right) f^{\prime}\left(\phi^{t}\right)$

$$
\begin{aligned}
& +\frac{1}{6} \Delta t^{3} f\left(\phi^{t}\right)\left\{f^{\prime}\left(\phi^{t}\right)\right\}^{2} \\
& +\frac{1}{6} \Delta t^{3}\left\{f\left(\phi^{t}\right)\right\}^{2} f^{\prime \prime}\left(\phi^{t}\right)+O\left(\Delta t^{4}\right) .
\end{aligned}
$$

This shows that the three-step scheme used in this model only has second-order accuracy, despite the three steps. However, it has third-order accuracy for linear equations, because $f^{\prime \prime}(\phi)=0$ if $f(\phi)$ is a linear function. This scheme can calculate small-amplitude perturbations with third-order accuracy, and finite-amplitude waves with second-order accuracy. In terms of numerical stability, this scheme has a stability region, which is almost the same as in standard explicit RK schemes with three steps for small perturbations. The stability region is wider than for RK schemes with secondorder accuracy, and includes the imaginary axis, which corresponds to neutral rotating modes, while that for secondorder schemes does not. This is why we choose the three-step method instead of the two-step method.

The advantage of this scheme compared with ordinary three-step schemes is the reduction of memory load and storage, which is one of the most expensive components of recent computers with low byte per flop (B / F) ratios, along with the benefit of higher numerical stability.

\section{A2.2 Physical processes}

The acoustic wave is the fastest mode in the dynamical processes, and the temporal interval for dynamical processes must be less than the grid spacing divided by the speed of the acoustic wave, to satisfy the Courant-Friedrichs-Lewy (CFL) condition. However, the timescale of the physical processes is usually much longer than the interval, so the temporal interval for the physical processes can be much longer than for the dynamical processes. We use a larger temporal interval to calculate the tendencies of the physical processes than of the dynamical processes. We call the time step for the physical processes a large time step, and for dynamical processes, a small time step.

Traditionally, tendencies in physical processes are calculated with large time steps, and the prognostic variables are updated with the Euler scheme with the tendency for large time steps. This causes an artificial acoustic wave, as described below. In this model, the tendency in some physical processes, such as surface flux (sensible heat flux and latent heat flux) and eddy viscosity and diffusion, are calculated with large time steps in the same way, but they are added to the prognostic variables with the tendency of dynamical processes in small time steps.

Figure A2 shows the horizontal averaged vertical velocity in the planetary boundary layer experiment, whose details are described in Sect. 3. In the control run, in which the temporal interval for the large time step ( $\left.\Delta t_{\text {phys }}\right)$ is the same as that for the small time step $\left(\Delta t_{\mathrm{dyn}}\right)$, the velocity is almost zero, as shown in Fig. A2a. However, in the case where $\Delta t_{\text {phys }}>$ $\Delta t_{\text {dyn }}$ and the tendency of the surface flux is added to the 


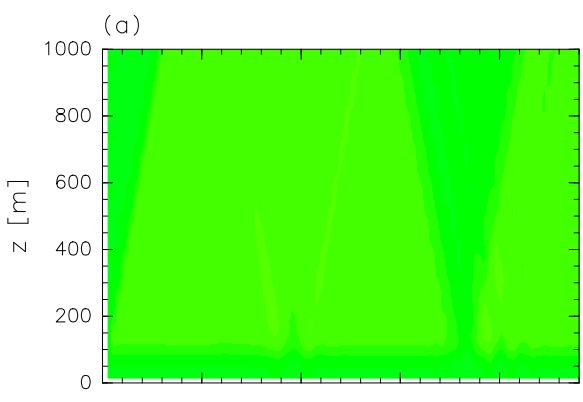

(b)
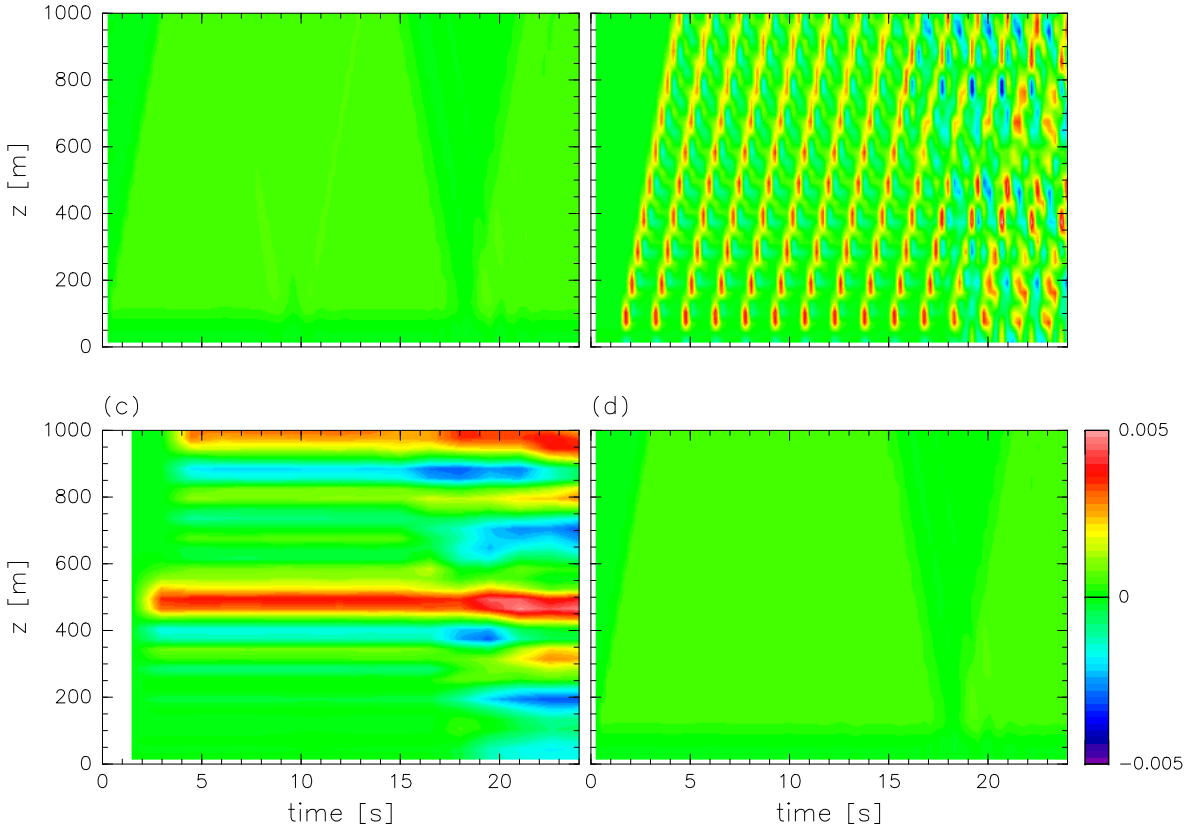

Figure A2. Time-height section of horizontal mean vertical velocity in runs where (a) $\Delta t_{\text {dyn }}=\Delta t_{\text {phys }}=0.03 \mathrm{~s}$ and $\Delta t_{\text {output }}=0.3 \mathrm{~s}$, (b) $\Delta t_{\text {dyn }}=0.03 \mathrm{~s}, \Delta t_{\text {phys }}=1.5 \mathrm{~s}$, and $\Delta t_{\text {output }}=0.3 \mathrm{~s}$, and surface flux is added at large time step intermittently, (c) same as (b) but $\Delta t_{\text {output }}=\Delta t_{\text {phys }}=1.5 \mathrm{~s}$, and (d) same as (b) but surface flux is added at small time step.

prognostic variables at the large time step as in the traditional manner, a vertically propagating artificial wave can be seen, as in Fig. A2b.

In addition, such a wave radiating periodically from a fixed location can cause a spurious stationary wave in the simulation output. This could lead to misinterpretation of the simulation results, although this problem is not directly based on physics or modeling. In most practical cases, the temporal interval for historical output ( $\left.\Delta t_{\text {output }}\right)$ is a multiple of $\Delta t_{\text {large }}$, and snapshots of physical quantities are the output. In such cases, every snapshot has artificial acoustic waves with the same phase, because the sampling frequency is not sufficient. This results in a spurious stationary wave in the historical output, as we can see in Fig. A2c, that is, forcing by physical processes added at small frequencies results in a spurious stationary wave in the historical data.

The artificial acoustic wave and resulting spurious stationary wave can be avoided if the prognostic variables are updated with the tendency calculated in the physical processes with a small time step, although the tendency is calculated with a large time step. Figure A2d shows that the artificial acoustic wave in panels $\mathrm{b}$ and $\mathrm{c}$ does not appear with this method. This shows that the tendency of the physical processes can be calculated with a large time step (i.e., not every small time step), while the time integration must be done with the dynamical process at a small time step.

\section{A3 Spatial discretization for the dynamical processes}

We employ the Arakawa-C staggered grid. Central difference schemes are used for the spatial differential for the dynamical processes, because waves such as gravity waves and acoustic waves generally cannot keep their spatial symmetry with odd-order schemes. Based on a consideration of numerical stability (see Appendix B), we choose the fourth-order central difference scheme for the advection (or convection) terms, and the second-order central difference scheme for the pressure gradient term in the momentum equations and divergence term in the continuity equation.

Before the discretization of the differential equations, we diagnose several quantities from the prognostic variables.

\section{Full-level pressure and potential temperature}

$$
\begin{aligned}
p_{i, j, k} & =p_{00}\left[\frac{(\rho \theta)_{i, j, k} R^{*}}{p_{00}}\right]^{\frac{c_{p}^{*}}{c_{p}^{*}-R^{*}}}, \\
\theta_{i, j, k} & =\frac{(\rho \theta)_{i, j, k}}{\rho_{i, j, k}} .
\end{aligned}
$$

\section{Half-level density}

$$
\begin{aligned}
\bar{\rho}_{i+\frac{1}{2}, j, k} & =\frac{\rho_{i+1, j, k}+\rho_{i, j, k}}{2}, \\
\bar{\rho}_{i, j+\frac{1}{2}, k} & =\frac{\rho_{i, j+1, k}+\rho_{i, j, k}}{2},
\end{aligned}
$$


$\bar{\rho}_{i, j, k+\frac{1}{2}}=\frac{\rho_{i, j, k+1}+\rho_{i, j, k}}{2}$.

\section{Half-level velocity}

$\bar{u}_{i+\frac{1}{2}, j, k}=\frac{(\rho u)_{i+\frac{1}{2}, j, k}}{\bar{\rho}_{i+\frac{1}{2}, j, k}}$,

$\bar{v}_{i, j+\frac{1}{2}, k}=\frac{(\rho v)_{i, j+\frac{1}{2}, k}}{\bar{\rho}_{i, j+\frac{1}{2}, k}}$,

$\bar{w}_{i, j, k+\frac{1}{2}}=\frac{(\rho w)_{i, j, k+\frac{1}{2}}}{\bar{\rho}_{i, j, k+\frac{1}{2}}}$.

\section{Full-level velocity}

$\bar{u}_{i, j, k}=\frac{(\rho u)_{i+\frac{1}{2}, j, k}+(\rho u)_{i-\frac{1}{2}, j, k}}{2 \rho_{i, j, k}}$,

$\bar{v}_{i, j, k}=\frac{(\rho v)_{i, j+\frac{1}{2}, k}+(\rho v)_{i, j-\frac{1}{2}, k}}{2 \rho_{i, j, k}}$,

$\bar{w}_{i, j, k}=\frac{(\rho w)_{i, j, k+\frac{1}{2}}+(\rho w)_{i, j, k-\frac{1}{2}}}{2 \rho_{i, j, k}}$.

\section{A3.1 Continuity equation}

Divergence in the continuity equation is calculated with the second-order central difference scheme. The continuity equation is discretized as

$$
\begin{aligned}
\left.\frac{\partial \rho}{\partial t}\right|_{i, j, k}= & -\frac{(\rho u)_{i+\frac{1}{2}, j, k}-(\rho u)_{i-\frac{1}{2}, j, k}}{\Delta x_{i}} \\
& -\frac{(\rho v)_{i, j+\frac{1}{2}, k}-(\rho v)_{i, j-\frac{1}{2}, k}}{\Delta y_{j}} \\
& -\frac{(\rho w)_{i, j, k+\frac{1}{2}}-(\rho w)_{i, j, k-\frac{1}{2}}}{\Delta z_{k}} .
\end{aligned}
$$

\section{A3.2 Momentum equations}

The advection terms and pressure gradient term are calculated with the fourth- and second-order central difference schemes, respectively. The momentum equation is discretized as

$$
\begin{aligned}
& \left.\frac{\partial \rho u}{\partial t}\right|_{i+\frac{1}{2}, j, k}=-\frac{{\overline{(\rho u)_{i+1, j, k}}}_{\bar{u}_{i+1, j, k}}-{\overline{(\rho u)_{i, j, k}}}_{i, j, k}}{\Delta x_{i+\frac{1}{2}}}
\end{aligned}
$$

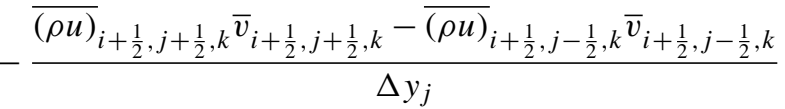

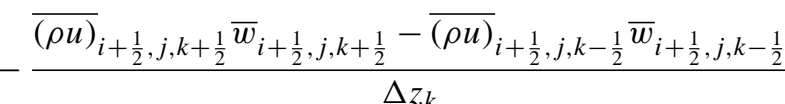

$$
\begin{aligned}
& -\frac{p_{i+1, j, k}-p_{i, j, k}}{\Delta x_{i+\frac{1}{2}}},
\end{aligned}
$$

where

$$
\begin{aligned}
& \overline{(\rho u}_{i, j, k}=\frac{-(\rho u)_{i+\frac{3}{2}, j, k}+7(\rho u)_{i+\frac{1}{2}, j, k}}{12} \\
& +\frac{7(\rho u)_{i-\frac{1}{2}, j, k}-(\rho u)_{i-\frac{3}{2}, j, k}}{12},
\end{aligned}
$$

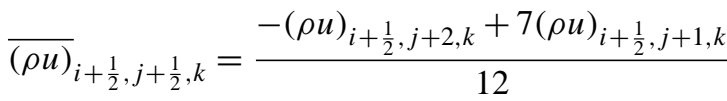

$$
\begin{aligned}
& +\frac{7(\rho u)_{i+\frac{1}{2}, j, k}-(\rho u)_{i+\frac{1}{2}, j-1, k}}{12},
\end{aligned}
$$

$$
\begin{aligned}
{\overline{(\rho u)_{i+\frac{1}{2}, j, k+\frac{1}{2}}}}= & \frac{-(\rho u)_{i+\frac{1}{2}, j, k+2}+7(\rho u)_{i+\frac{1}{2}, j, k+1}}{12} \\
& +\frac{7(\rho u)_{i+\frac{1}{2}, j, k}-(\rho u)_{i+\frac{1}{2}, j, k-1}}{12},
\end{aligned}
$$

and the velocities at the cell wall for the staggered control volume in the $x$ direction are defined as

$$
\begin{aligned}
\bar{v}_{i+\frac{1}{2}, j+\frac{1}{2}, k} & =\frac{\bar{v}_{i, j+\frac{1}{2}, k}+\bar{v}_{i+1, j+\frac{1}{2}, k}}{2}, \\
\bar{w}_{i+\frac{1}{2}, j, k+\frac{1}{2}} & =\frac{\bar{w}_{i, j, k+\frac{1}{2}}+\bar{w}_{i+1, j, k+\frac{1}{2}}}{2} .
\end{aligned}
$$

In this form, the fourth-order accuracy in the advection term is guaranteed on the condition of constant velocity. The momentum equations in the $y$ and $z$ directions are discretized in the same way. In the vertical equation, $\bar{\rho}_{i, j, k+\frac{1}{2}} g$ is added.

\section{A3.3 Thermodynamics equation}

The thermodynamics equation is discretized as

$$
\begin{gathered}
\left.\frac{\partial \rho \theta}{\partial t}\right|_{i, j, k}=-\frac{(\rho u)_{i+\frac{1}{2}, j, k} \bar{\theta}_{i+\frac{1}{2}, j, k}-(\rho u)_{i-\frac{1}{2}, j, k} \bar{\theta}_{i-\frac{1}{2}, j, k}}{\Delta x_{i}} \\
-\frac{(\rho v)_{i, j+\frac{1}{2}, k} \bar{\theta}_{i, j+\frac{1}{2}, k}-(\rho v)_{i, j-\frac{1}{2}, k} \bar{\theta}_{i, j-\frac{1}{2}, k}}{\Delta y_{j}} \\
-\frac{(\rho w)_{i, j, k+\frac{1}{2}} \bar{\theta}_{i, j, k+\frac{1}{2}}-(\rho w)_{i, j, k-\frac{1}{2}} \bar{\theta}_{i, j, k-\frac{1}{2}}}{\Delta z_{k}},
\end{gathered}
$$

where

$\bar{\theta}_{i+\frac{1}{2}, j, k}=\frac{-\theta_{i+2, j, k}+7 \theta_{i+1, j, k}+7 \theta_{i, j, k}-\theta_{i-1, j, k}}{12}$,

and $\bar{\theta}_{i, j+\frac{1}{2}, k}$, and $\bar{\theta}_{i, j, k+\frac{1}{2}}$ are defined in the same way.

\section{A3.4 Tracer advection}

The tracer advection process is done with the Euler scheme after the temporal integration of the dynamical variables $(\rho$, 
$\rho u, \rho v, \rho w$, and $\rho \theta)$. We impose the consistency with continuity (CWC; Gross et al., 2002) and monotonicity of the tracer advection following Niwa et al. (2011).

With the condition of no source / sink, the ratio of the mass of tracers to the total mass in the advection process should be conserved along the trajectory. It is, at the least, necessary that the spatially constant mass concentration should be kept in any motion of fluid. In order to satisfy this condition, we use the same mass flux at the last Runge-Kutta process $\left(k_{3} / \Delta t\right.$ in Eq. A72) for integration of tracers:

$$
\begin{array}{r}
\frac{(\rho q)_{i, j, k}^{t+\Delta t}-(\rho q)_{i, j, k}^{t}}{\Delta t}= \\
-\frac{(\rho u)_{i+\frac{1}{2}, j, k} \bar{q}_{i+\frac{1}{2}, j, k}-(\rho u)_{i-\frac{1}{2}, j, k} \bar{q}_{i-\frac{1}{2}, j, k}}{\Delta x_{i}} \\
-\frac{(\rho v)_{i, j+\frac{1}{2}, k} \bar{q}_{i, j+\frac{1}{2}, k}-(\rho v)_{i, j-\frac{1}{2}, k} \bar{q}_{i, j-\frac{1}{2}, k}}{\Delta y_{j}} \\
-\frac{(\rho w)_{i, j, k+\frac{1}{2}} \bar{q}_{i, j, k+\frac{1}{2}}-(\rho w)_{i, j, k-\frac{1}{2}} \bar{q}_{i, j, k-\frac{1}{2}}}{\Delta z_{k}}
\end{array}
$$

In order to satisfy the monotonicity of tracer advection, we employ the flux-corrected transport (FCT) scheme, which is a hybrid scheme with a higher-order difference scheme and first-order upwind scheme (Zalesak, 1979). In this model, we use the fourth-order central difference scheme as a higherorder scheme.

If the fourth-order central difference is applied, $\bar{q}$ is discretized as

$\bar{q}_{i+\frac{1}{2}, j, k}^{\text {high }}=$

$$
\frac{-q_{i+2, j, k}+7 q_{i+1, j, k}+7 q_{i, j, k}-q_{i-1, j, k}}{12},
$$

and $\bar{q}_{i, j+\frac{1}{2}, k}^{\text {high }}$ and $\bar{q}_{i, j, k+\frac{1}{2}}^{\text {high }}$ are defined in the same way. On the other hand, in the first-order upwind scheme, $\bar{q}$ is described as

$\bar{q}_{i+\frac{1}{2}, j, k}^{\text {low }}= \begin{cases}q_{i, j, k} & \text { if }(\rho u)_{i+\frac{1}{2}, j, k}>0, \\ q_{i+1, j, k} & \text { otherwise, }\end{cases}$

and $\bar{q}_{i, j+\frac{1}{2}, k}^{\text {low }}$ and $\bar{q}_{i, j, k+\frac{1}{2}}^{\text {low }}$ are defined in the same way. The actual $\bar{q}$ is described as

$$
\begin{aligned}
\bar{q}_{i+\frac{1}{2}, j, k} & =C_{i+\frac{1}{2}, j, k} \bar{q}_{i+\frac{1}{2}, j, k}^{\text {high }} \\
& +\left(1-C_{i+\frac{1}{2}, j, k}\right) \bar{q}_{i+\frac{1}{2}, j, k}^{\text {low }},
\end{aligned}
$$

and $\bar{q}_{i, j+\frac{1}{2}, k}$ and $\bar{q}_{i, j, k+\frac{1}{2}}$ are defined in the same way.

Equation (A95) can be written as

$$
(\rho q)_{i, j, k}^{n+1}=(\rho q)_{i, j, k}^{n}-\frac{1}{\Delta x_{i} \Delta y_{j} \Delta z_{k}}[
$$

$$
\begin{aligned}
& +\left[C_{i+\frac{1}{2}, j, k} F_{i+\frac{1}{2}, j, k}^{\text {high }}+\left(1-C_{i+\frac{1}{2}, j, k}\right) F_{i+\frac{1}{2}, j, k}^{\text {low }}\right] \\
& -\left[C_{i-\frac{1}{2}, j, k} F_{i-\frac{1}{2}, j, k}^{\text {high }}+\left(1-C_{i-\frac{1}{2}, j, k}\right) F_{i-\frac{1}{2}, j, k}^{\text {low }}\right] \\
& +\left[C_{i, j+\frac{1}{2}, k} F_{i, j+\frac{1}{2}, k}^{\text {high }}+\left(1-C_{i, j+\frac{1}{2}, k}\right) F_{i, j+\frac{1}{2}, k}^{\text {low }}\right] \\
& -\left[C_{i, j-\frac{1}{2}, k} F_{i, j-\frac{1}{2}, k}^{\text {high }}+\left(1-C_{i, j-\frac{1}{2}, k}\right) F_{i, j-\frac{1}{2}, k}^{\text {low }}\right] \\
& +\left[C_{i, j, k+\frac{1}{2}} F_{i, j, k+\frac{1}{2}}^{\text {high }}+\left(1-C_{i, j, k+\frac{1}{2}}\right) F_{i, j, k+\frac{1}{2}}^{\text {low }}\right] \\
& \left.-\left[C_{i, j, k-\frac{1}{2}} F_{i, j, k-\frac{1}{2}}^{\text {high }}+\left(1-C_{i, j, k-\frac{1}{2}}\right) F_{i, j, k-\frac{1}{2}}^{\text {low }}\right]\right],
\end{aligned}
$$

where

$F_{i+\frac{1}{2}, j, k}^{\text {high,low }}=\Delta t \Delta y_{j} \Delta z_{k}(\rho u)_{i+\frac{1}{2}, j, k} q_{i+\frac{1}{2}, j, k}^{\text {high,low }}$,

and $F_{i, j+\frac{1}{2}, k}^{\text {high,low }}$ and $F_{i, j, k+\frac{1}{2}}^{\text {high,low }}$ are defined in the same way. The anti-diffusive flux is defined as

$$
A_{i+\frac{1}{2}, j, k}=F_{i+\frac{1}{2}, j, k}^{\text {high }}-F_{i+\frac{1}{2}, j, k}^{\text {low }},
$$

and $A_{i, j+\frac{1}{2}, k}$ and $A_{i, j, k+\frac{1}{2}}$ are defined in the same way. Equation (A99) can be rewritten as

$$
\begin{aligned}
(\rho q)_{i, j, k}^{n+1}= & (\rho q)_{i, j, k}^{n}-\frac{1}{\Delta x_{i} \Delta y_{j} \Delta z_{k}}\{ \\
& +\left[F_{i+\frac{1}{2}, j, k}^{\text {low }}+C_{i+\frac{1}{2}, j, k} A_{i+\frac{1}{2}, j, k}\right] \\
& -\left[F_{i-\frac{1}{2}, j, k}^{\text {low }}+C_{i-\frac{1}{2}, j, k} A_{i-\frac{1}{2}, j, k}\right] \\
& +\left[F_{i, j+\frac{1}{2}, k}^{\text {low }}+C_{i, j+\frac{1}{2}, k} A_{i, j+\frac{1}{2}, k}\right] \\
& -\left[F_{i, j-\frac{1}{2}, k}^{\text {low }}+C_{i, j-\frac{1}{2}, k} A_{i, j-\frac{1}{2}, k}\right] \\
& +\left[F_{i, j, k+\frac{1}{2}}^{\text {low }}+C_{i, j, k+\frac{1}{2}} A_{i, j, k+\frac{1}{2}}\right] \\
& \left.-\left[F_{i, j, k-\frac{1}{2}}^{\text {low }}+C_{i, j, k-\frac{1}{2}} A_{i, j, k-\frac{1}{2}}\right]\right\}
\end{aligned}
$$

In practice, we calculate Eq. (A102) by the following steps:

1. The tentative values are calculated using the low-order flux:

$$
\begin{aligned}
(\rho q)_{i, j, k}^{\dagger}= & (\rho q)_{i, j, k}^{n} \\
& -\frac{1}{\Delta x_{i} \Delta y_{j} \Delta z_{k}}\left[F_{i+\frac{1}{2}, j, k}^{\text {low }}-F_{i-\frac{1}{2}, j, k}^{\text {low }}\right. \\
& \quad+F_{i, j+\frac{1}{2}, k}^{\text {low }}-F_{i, j-\frac{1}{2}, k}^{\text {low }} \\
& \left.\quad F_{i, j, k+\frac{1}{2}}^{\text {low }}-F_{i, j, k-\frac{1}{2}}^{\text {low }}\right]
\end{aligned}
$$


2. Allowable maximum and minimum values are calculated:

$$
\begin{aligned}
(\rho q)_{i, j, k}^{\max }= & \max \{ \\
& \max \left[(\rho q)_{i, j, k}^{\dagger},(\rho q)_{i, j, k}^{n}\right], \\
& \max \left[(\rho q)_{i-1, j, k}^{\dagger},(\rho q)_{i-1, j, k}^{n}\right] \\
& \max \left[(\rho q)_{i+1, j, k}^{\dagger},(\rho q)_{i+1, j, k}^{n}\right] \\
& \max \left[(\rho q)_{i, j-1, k}^{\dagger},(\rho q)_{i, j-1, k}^{n}\right] \\
& \max \left[(\rho q)_{i, j+1, k}^{\dagger},(\rho q)_{i, j+1, k}^{n}\right] \\
& \max \left[(\rho q)_{i, j, k-1}^{\dagger},(\rho q)_{i, j, k-1}^{n}\right] \\
& \left.\max \left[(\rho q)_{i, j, k+1}^{\dagger},(\rho q)_{i, j, k+1}^{n}\right]\right\} \\
(\rho q)_{i, j, k} \min ^{\dagger} & \min \{ \\
& \min \left[(\rho q)_{i, j, k}^{\dagger},(\rho q)_{i, j, k}^{n}\right], \\
& \min \left[(\rho q)_{i-1, j, k}^{\dagger},(\rho q)_{i-1, j, k}^{n}\right] \\
& \min \left[(\rho q)_{i+1, j, k}^{\dagger},(\rho q)_{i+1, j, k}^{n}\right], \\
& \min \left[(\rho q)_{i, j-1, k}^{\dagger},(\rho q)_{i, j-1, k}^{n}\right] \\
& \min \left[(\rho q)_{i, j+1, k}^{\dagger},(\rho q)_{i, j+1, k}^{n}\right], \\
& \min \left[(\rho q)_{i, j, k-1}^{\dagger},(\rho q)_{i, j, k-1}^{n}\right] \\
& \left.\min \left[(\rho q)_{i, j, k+1}^{\dagger},(\rho q)_{i, j, k+1}^{n}\right]\right\}
\end{aligned}
$$

3. Several values for the flux limiter are calculated:

$$
\begin{gathered}
P_{i, j, k}^{+}=-\min \left(0, A_{i+\frac{1}{2}, j, k}\right)+\max \left(0, A_{i-\frac{1}{2}, j, k}\right) \\
-\min \left(0, A_{i, j+\frac{1}{2}, k}\right)+\max \left(0, A_{i, j-\frac{1}{2}, k}\right) \\
-\min \left(0, A_{i, j, k+\frac{1}{2}}\right)+\max \left(0, A_{i, j, k-\frac{1}{2}}\right), \\
P_{i, j, k}^{-}=\max \left(0, A_{i+\frac{1}{2}, j, k}\right)-\min \left(0, A_{i-\frac{1}{2}, j, k}\right) \\
\max \left(0, A_{i, j+\frac{1}{2}, k}\right)-\min \left(0, A_{i, j-\frac{1}{2}, k}\right) \\
\max \left(0, A_{i, j, k+\frac{1}{2}}\right)-\min \left(0, A_{i, j, k-\frac{1}{2}}\right), \\
Q_{i, j, k}^{+}=\left[(\rho q)_{i, j, k}^{\max }-(\rho q)_{i, j, k}^{\dagger}\right] \Delta x_{i} \Delta y_{j} \Delta z_{k}, \\
Q_{i, j, k}^{-}=\left[(\rho q)_{i, j, k}^{\dagger}-(\rho q)_{i, j, k}^{\min }\right] \Delta x_{i} \Delta y_{j} \Delta z_{k}, \\
R_{i, j, k}^{+}=\left\{\begin{array} { l l } 
{ \operatorname { m i n } ( 1 , Q _ { i , j , k } ^ { + } / P _ { i , j , k } ^ { + } ) } & { \text { if } P _ { i , j , k } ^ { + } > 0 , } \\
{ 0 } & { \text { if } P _ { i , j , k } ^ { + } = 0 , }
\end{array} \left(\mathrm{A},{ }^{+}, P_{i, j, k}>0,\right.\right.
\end{gathered}
$$

4. The flux limiters at the cell wall are calculated:

$$
\begin{aligned}
& C_{i+\frac{1}{2}, j, k}= \\
& \begin{cases}\min \left(R_{i+1, j, k}^{+}, R_{i, j, k}^{-}\right) & \text {if } A_{i+\frac{1}{2}, j, k} \geq 0, \\
\min \left(R_{i, j, k}^{+}, R_{i+1, j, k}^{-}\right) & \text {if } A_{i+\frac{1}{2}, j, k}<0,\end{cases}
\end{aligned}
$$

and $C_{i, j+\frac{1}{2}, k}$ and $C_{i, j, k+\frac{1}{2}}$ are defined as the same way.

\section{A3.5 Boundary condition}

The boundary condition at the top and bottom boundaries is

$$
\begin{aligned}
& w_{i, j, k_{\max }+\frac{1}{2}}=w_{i, j, k_{\min }-\frac{1}{2}}=0, \\
& (\rho w)_{i, j, k_{\max }+\frac{1}{2}}=(\rho w)_{i, j, k_{\min }-\frac{1}{2}}=0, \\
& \left(\rho q_{\mathrm{v}} w\right)_{i, j, k_{\max }+\frac{1}{2}}=\left(\rho q_{\mathrm{v}} w\right)_{i, j, k_{\min }-\frac{1}{2}}=0, \\
& \left(\rho q_{1} w\right)_{i, j, k_{\max }+\frac{1}{2}}=\left(\rho q_{1} w\right)_{i, j, k_{\min }-\frac{1}{2}}=0, \\
& \left(\rho q_{\mathrm{s}} w\right)_{i, j, k_{\max }+\frac{1}{2}}=\left(\rho q_{\mathrm{s}} w\right)_{i, j, k_{\min }-\frac{1}{2}}=0, \\
& (\rho u w)_{i, j, k_{\max }+\frac{1}{2}}=(\rho u w)_{i, j, k_{\min }-\frac{1}{2}}=0, \\
& (\rho v w)_{i, j, k_{\max }+\frac{1}{2}}=(\rho v w)_{i, j, k_{\min }-\frac{1}{2}}=0, \\
& (\rho \theta)_{i, j, k_{\max }+\frac{1}{2}}=(\rho \theta)_{i, j, k_{\min }-\frac{1}{2}}=0 . \\
& w_{i, j, k_{\max }+\frac{1}{2}}=0, \\
& w_{i, j, k_{\min }-\frac{1}{2}}=0 .
\end{aligned}
$$

This leads to the boundary condition of the vertical momentum:

$$
\begin{aligned}
& (\rho w)_{i, j, k_{\max }+\frac{1}{2}}=0, \\
& (\rho w)_{i, j, k_{\min }-\frac{1}{2}}=0 .
\end{aligned}
$$

For other prognostic variables, the vertical fluxes at the top and bottom boundaries are zero, except those from physical processes.

\section{A4 Numerical filter}

We impose an explicit numerical filter using the numerical viscosity and diffusion. Although the filter is necessary for numerical stability, too strong a filter could dampen down any physically meaningful variability. In this subsection, we describe the numerical filters used in this model, and discuss the strength of the filter.

In order to damp down the higher wave number component selectively, we adopt the hyperviscosity and diffusion in the traditional way. The hyperviscosity and diffusion of the $n$th order is defined as

$\frac{\partial}{\partial x}\left[v \rho \frac{\partial^{n-1} f}{\partial x^{n-1}}\right]$,

where $f$ is an arbitrary variable $(f \in \rho, u, v, w, \theta, q)$. 
The Laplacian of $f$ is discretized as

$$
\begin{aligned}
\Delta f_{i} & =\frac{1}{\Delta x_{i}}\left[\frac{1}{\Delta x_{i+\frac{1}{2}}} f_{i+1}-\left(\frac{1}{\Delta x_{i+\frac{1}{2}}}+\frac{1}{\Delta x_{i-\frac{1}{2}}}\right) f_{i}\right. \\
& \left.+\frac{1}{\Delta x_{i-\frac{1}{2}}} f_{i-1}\right],
\end{aligned}
$$

and

$$
\begin{aligned}
\Delta^{n / 2} f_{i}= & \frac{1}{\Delta x_{i}}\left[\frac{1}{\Delta x_{i+\frac{1}{2}}} \Delta^{n / 2-1} f_{i+1}\right. \\
& -\left(\frac{1}{\Delta x_{i+\frac{1}{2}}}+\frac{1}{\Delta x_{i-\frac{1}{2}}}\right) \Delta^{n / 2-1} f_{i} \\
& \left.+\frac{1}{\Delta x_{i-\frac{1}{2}}} \Delta^{n / 2-1} f_{i-1}\right] .
\end{aligned}
$$

Here we consider spatially dependent grid interval in calculating the Laplacian. If it is calculated with constant $\Delta x_{i}$ as

$$
\begin{aligned}
\Delta f_{i}= & \frac{1}{\Delta x_{i}^{2}}\left(f_{i+1}-2 f_{i}+f_{i-1}\right), \\
\Delta^{n / 2} f_{i} & =\frac{1}{\Delta x_{i}^{2}}\left(\Delta^{n / 2-1} f_{i+1}-2 \Delta^{n / 2-1} f_{i}\right. \\
& \left.+\Delta^{n / 2-1} f_{i-1}\right),
\end{aligned}
$$

non-negligible numerical noise appears where the grid spacing varies (e.g., stretching layer near the top boundary).

The hyperviscosity and diffusion can be discretized as

$\frac{\partial}{\partial x}\left[v \rho \frac{\partial^{n-1} f}{\partial^{n-1} x}\right] \sim \frac{F_{i+\frac{1}{2}}-F_{i-\frac{1}{2}}}{\Delta x_{i}}$,

where

$F_{i+\frac{1}{2}} \frac{\nu_{i+\frac{1}{2}} \rho_{i+\frac{1}{2}}}{\Delta x_{i+\frac{1}{2}}}\left(\Delta^{n / 2-1} f_{i+1}-\Delta^{n / 2-1} f_{i}\right)$.

The coefficient, $v$, is written as

$v_{i+\frac{1}{2}}=(-1)^{n / 2+1} \gamma \frac{\Delta x_{i+\frac{1}{2}}^{n}}{2^{n} \Delta t}$,

where $\gamma$ is a non-dimensional coefficient. One-dimensional sinusoidal two-grid noise will decay to $1 / e$ with $1 / \gamma$ time steps. Note that the theoretical $e$-folding time is $\frac{2^{n}}{\pi^{n}} \frac{\Delta t}{\gamma}$. However, it is $\frac{\Delta t}{\gamma}$ with the fourth-order central scheme used in this model.

For the numerical stability of the numerical filter itself, it should satisfy

$\gamma<1$

for the one-dimensional two-grid noise, and

$\gamma<\frac{1}{3}$ for the three-dimensional two-grid noise. The conditions might be stricter for other types of noise.

The flux, $F$, for the numerical filter is added to the advective flux as

$(\rho u f)_{i+\frac{1}{2}}^{\dagger}=(\rho u f)_{i+\frac{1}{2}}+F_{i+\frac{1}{2}}$,

where the first term of the right-hand side is the flux calculated by the advection scheme. In the present model, the advection scheme is the fourth-order central difference scheme (see Appendix A3). This concept is very important for the CWC condition in the tracer equations (see Appendix A3.4). The modified mass flux of the numerical filter should be used in the tracer advection; otherwise, the CWC condition is violated.

The numerical viscosity and diffusion in the $y$ and $z$ directions are formulated in the same way as in the $x$ direction, although a special treatment for the $z$ direction is needed. At the top and bottom boundaries, the flux must be zero, $F_{k_{\max }+\frac{1}{2}}=F_{k_{\min }-\frac{1}{2}}=0$. In order to calculate the $F_{k_{\max }-\frac{1}{2}}$ and $F_{k_{\min }+\frac{1}{2}}$, values beyond the boundaries, $f_{k_{\max }+1}$ and $f_{k_{\min }-1}$, are required, then the mirror boundary condition is assumed: $f_{k_{\max }+1}=-f_{k_{\max }}$ and $f_{k_{\min }-1}=-f_{k_{\min }}$. This condition is appropriate to cause the decay the vertical two-grid noise.

Vertical profiles of density, potential temperature, and water vapor usually have significant (e.g., logarithmic) dependencies on height. Equation (A130) has a non-zero value even for the steady state, and the numerical filter produces artificial motion. To reduce this artificial motion, we introduce a reference profile which is a function of height, and deviation from the reference is used as $f$ instead of $\rho, \theta$, and $q_{\mathrm{v}}$ in calculating the numerical filter. The reference profile can be chosen arbitrarily, but a profile under hydrostatic balance is usually chosen.

Determination of the value of the non-dimensional coefficient $\gamma$ is an important issue. If it is too small, the simulation could be numerically unstable or numerical noise could violate the physical variability, while variability could be dampened down too much if $\gamma$ is too large. At least, the effect of the numerical filter on the phenomena of the scale we want to simulate must be reasonably smaller than the effect of the physically oriented viscosity and diffusion, i.e., the eddy viscosity and diffusion representing the effect of the SGS turbulence.

The $e$-folding time of an eddy whose spatial scale is $L$ is $\frac{2^{n} L^{n}}{\gamma \Delta x^{n}} \Delta t$ according to the numerical filter, and is $\frac{L^{2}}{C_{\mathrm{S}}^{2}(2 \Delta x)^{2}|S|}$ by eddy viscosity and diffusion for an isotropic grid under neutral stability conditions. The $e$-folding time of an eddy whose scale is larger than the effective resolution of the numerical filter should be longer than that for eddy viscosity and diffusion, where the effective resolution is the smallest scale of physically meaningful phenomena that a simulation can represent. It requires that $\gamma<2^{n+2} C_{\mathrm{S}}^{2}|S| \Delta t \frac{L^{n-2}}{\Delta x^{n-2}}$. The magnitude of the characteristic velocity, $U$, is assumed 
to be $O(1)-O(10) \mathrm{m} \mathrm{s}^{-1}$ for motions whose spatial scale is approximately equal to the effective resolution. Then, $|S| \Delta t\left(\sim \frac{U}{\Delta x} \Delta t\right)$ is estimated to be $O\left(10^{-3}\right)-O\left(10^{-2}\right)$, because $\Delta x / \Delta t$ is $O(350)-O(1000) \mathrm{m} \mathrm{s}^{-1}$ according to the CFL condition. Roughly speaking, the effective resolution is usually several times the grid spacing. Here, we assume $L / \Delta x \sim 2-10$; Skamarock (2004) suggested that the effective resolution is about 6 times the grid spacing. It is found that $\gamma$ should be

$\gamma<O\left(10^{-3}\right)-O(1)$ for $n=4$,

$\gamma<O(1)-O\left(10^{5}\right)$ for $n=8$.

This condition is stricter than the stability condition Eq. (A134) for $n=4$, so this condition must be considered when the value of $\gamma$ is determined if $n=4$ is chosen. On the other hand, it is always satisfied if the stability condition is satisfied for $n \geq 8$. This means that in this case, the effect of the numerical filter on phenomena whose spatial scale is larger than the effective resolution is always smaller than that of the eddy viscosity and diffusion, and this condition for $\gamma$ does not have to be considered if $n \geq 8$ is chosen. The advantage of an explicit numerical filter is that its strength can be controlled or tuned based on such physical considerations.

Odd-order advection schemes are generally more stable numerically than even-order schemes. In general, odd-order schemes can be divided into a central difference term and a filter term (and sometime other additional terms) conceptually. This implicit numerical filter stabilizes the calculations. We estimate the corresponding value of $\gamma$ for the implicit filter. The spatial differential of a scalar quantity $\phi$ with the ordinary third-order upwind difference scheme is written as

$$
\begin{gathered}
\left.\frac{\partial \phi}{\partial x}\right|_{i} \sim \frac{-\phi_{i+2}+8 \phi_{i+1}-8 \phi_{i-1}+\phi_{i-2}}{12 \Delta x}+\frac{\Delta x^{3}}{12} \\
|U| \frac{\phi_{i+2}-4 \phi_{i+1}+6 \phi_{i}-4 \phi_{i-1}+\phi_{i-2}}{\Delta x^{4}} .
\end{gathered}
$$

The first term on the right-hand side of the equation represents the central difference with fourth-order accuracy. The second term, divided by $|U|$, is written as

$$
\begin{gathered}
\frac{\Delta x^{3}}{12} \frac{\phi_{i+2}-4 \phi_{i+1}+6 \phi_{i}-4 \phi_{i-1}+\phi_{i-2}}{\Delta x^{4}} \\
\quad=\frac{\Delta x^{3}}{12}\left(\left.\frac{\partial^{4} \phi}{\partial x^{4}}\right|_{i}+O\left(\Delta x^{2}\right)\right),
\end{gathered}
$$

and corresponds to fourth-order hyperviscosity and diffusion. This can be considered as implicit numerical filter introduced to the scheme. Their dimensional coefficient is $|U| \frac{\Delta x^{3}}{12}$, and the non-dimensional coefficient is

$\gamma=|U| \frac{4 \Delta t}{3 \Delta x}$.

Considering the CFL condition, $\Delta t / \Delta x<1 / c, \gamma$ is $O\left(10^{-3}\right)-O\left(10^{-2}\right)$, if $U$ is assumed to be
$O(1)-O(10) \mathrm{m} \mathrm{s}^{-1}$, where $c$ is the speed of acoustic wave and is $O\left(10^{2}\right)-O\left(10^{3}\right)$. This mostly satisfies the necessary condition (Eq. A136).

The third-order scheme by Kawamura and Kuwahara (1984) is a modification of the third-order scheme to enable changing the strength of the numerical filter, and is written as

$$
\begin{gathered}
\left.\frac{\partial \phi}{\partial x}\right|_{i}=\frac{-\phi_{i+2}+8 \phi_{i+1}-8 \phi_{i-1}+\phi_{i-2}}{12 \Delta x}+C \frac{\Delta x^{3}}{4} \\
|U| \frac{\phi_{i+2}-4 \phi_{i+1}+6 \phi_{i}-4 \phi_{i-1}+\phi_{i-2}}{\Delta x^{4}} .
\end{gathered}
$$

We can change the constant $C$ to control the strength of the numerical filter.

Although these schemes can be thought of as being similar to central difference and explicit fourth-order numerical filters, the total coefficient of the filter depends on the velocity $U$. The strength of the implicit filter could be too large when the basic wind state is strong, and its effect is not always smaller than that of the eddy viscosity and diffusion representing the effect of SGS turbulence. As we discuss here, it is important to estimate the effect of the numerical filter used in a model, and to control or confirm that the artificial filter does not violate the viscosity and diffusion as a physical parameterization. This point is one of the most important factors in choosing a numerical scheme in this type of study.

\section{A5 Physical processes}

Currently, the following processes are implemented as physical processes in SCALE-LES.

- Cloud microphysics

- A one-moment three-category bulk scheme (Kessler, 1969)

- A one-moment six-category bulk scheme (Tomita, 2008)

- A two-moment six-category bulk scheme (Seiki and Nakajima, 2014)

- A bin method (Suzuki et al., 2010).

- Sub-grid turbulence

- A Smagorinsky-Lilly-type scheme including stability effect developed by Brown et al. (1994)

- A RANS (Reynolds-averaged Navier-Stokes) turbulence model (Nakanishi and Niino, 2004, level 2.5).

- Radiation

- A parallel plane radiation model (MstrnX; Sekiguchi and Nakajima, 2008).

- Surface flux 
- A Louis-type bulk model (Louis, 1979; Uno et al., 1995)

- A Beljaars-type bulk model (Beljaars and Holtslag, 1991; Wilson, 2001).

- Urban canopy

- A single-layer urban canopy model (Kusaka et al., 2001; Kusaka and Kimura, 2004).

\section{Appendix B: Numerical stability}

In this model, we use the second-order central difference scheme for the spatial differential terms of the pressure gradient and the divergence of mass flux, and the fourth-order scheme for advection terms. In this section, we investigate the numerical instability of the terms and show why we use the second-order scheme for these terms.

For simplicity, we assume a case in which the initial potential temperature is constant $\theta_{0}$, and the initial momentum is also constant. In addition, dry conditions are assumed. The potential temperature is to be constant at all times.

The governing equations are

$\frac{\partial \rho}{\partial t}=-\nabla \cdot(\rho \boldsymbol{u})$

$\frac{\partial \rho \boldsymbol{u}}{\partial t}=-\frac{\partial p}{\partial \boldsymbol{x}}$,

$p=p_{00}\left(\frac{\rho R_{\mathrm{d}} \theta_{0}}{p_{00}}\right)^{\frac{c_{p \mathrm{~d}}}{c_{v \mathrm{~d}}}}$.

To investigate the stability, the equations are linearized. The density is divided into the basic value and the deviation from the basic:

$\rho=\rho_{0}+\rho^{\prime}$,

where

$\rho_{0}=\frac{p_{00}}{R_{\mathrm{d}} \theta_{0}}$

Pressure is written as

$p \sim p_{00}\left(1+\frac{c_{p \mathrm{~d}}}{c_{v \mathrm{~d}}} \frac{\rho^{\prime}}{\rho_{0}}\right)=p_{00}+c^{2} \rho^{\prime}$,

where $c$ is the speed of the acoustic wave $\left(=\sqrt{\frac{c_{p \mathrm{~d}}}{c_{v \mathrm{~d}}} \frac{p_{00}}{\rho_{0}}}\right)$ in the reference state of $p=p_{00}$ and $\rho=\rho_{0}$.

The time derivatives are written as

$$
\begin{aligned}
\left.\frac{\partial \rho}{\partial t}\right|_{i, j, k} & =-\frac{-a(\rho u)_{i+1, j, k}+b(\rho u)_{i, j, k}-b(\rho u)_{i-1, j, k}+a(\rho u)_{i-2, j, k}}{(b-3 a) \Delta x} \\
& -\frac{-a(\rho v)_{i, j+1, k}+b(\rho v)_{i, j, k}-b(\rho v)_{i, j-1, k}+a(\rho v)_{i, j-2, k}}{(b-3 a) \Delta y} \\
& -\frac{-a(\rho w)_{i, j, k+1}+b(\rho w)_{i, j, k}-b(\rho w)_{i, j k-1}+a(\rho w)_{i, j k-2}}{(b-3 a) \Delta z}, \\
\left.\frac{\partial(\rho u)}{\partial t}\right|_{i, j, k} & =-c^{2} \frac{-a \rho_{i+2, j, k}+b \rho_{i+1, j, k}-b \rho_{i, j, k}+a \rho_{i-1, j, k}}{(b-3 a) \Delta x}, \\
\left.\frac{\partial(\rho v)}{\partial t}\right|_{i, j, k} & =-c^{2} \frac{-a \rho_{i, j+2, k}+b \rho_{i, j+1, k}-b \rho_{i, j, k}+a \rho_{i, j-1, k}}{(b-3 a) \Delta y}, \\
\left.\frac{\partial(\rho w)}{\partial t}\right|_{i, j, k} & =-c^{2} \frac{-a \rho_{i, j, k+2}+b \rho_{i, j, k+1}-b \rho_{i, j, k}+a \rho_{i, j, k-1}}{(b-3 a) \Delta z} .
\end{aligned}
$$

For the second-order central difference, $a=0$ and $b=1$, while $a=1$ and $b=9$ for the fourth order.

Now we consider the numerical stability of two-grid noise. An eigenanalysis showed that the two-grid noise is the most unstable eigenmode in all the cases we tested. The density with the noise can be written as $\rho_{i, j, k}^{t}=\rho_{0}+A \sin (\pi(i+j+$ $k)$ ). Using the third-step RK scheme, the density after one time step, $\rho_{i, j, k}^{t+\Delta t}$, is

$$
\begin{gathered}
\rho_{i, j, k}^{t+\Delta t}-\rho_{0}=\left(1-6 \frac{(a+b)^{2}}{(b-3 a)^{2}} c^{2} \frac{\Delta t^{2}}{\Delta x^{2}}\right) \\
\left(\rho_{i, j, k}^{t}-\rho_{0}\right) .
\end{gathered}
$$

The necessary condition for the two-grid noise to decrease in time is

$\left|1-6\left(\frac{a+b}{b-3 a} \frac{\Delta t}{\Delta t_{\mathrm{CFL}}}\right)^{2}\right|<1$,

then

$\Delta t<\frac{1}{\sqrt{3}} \Delta t_{\mathrm{CFL}}$

for the second-order spatial difference, and

$\Delta t<\frac{\sqrt{3}}{5} \Delta t_{\mathrm{CFL}}$

for the fourth order, where $\Delta t_{\mathrm{CFL}}=\Delta x / c$. If the fourthorder scheme is adopted, we need to make $\Delta t 0.6$ times (or number of time steps multiplied by 1.67) larger than that in the second-order scheme.

The accuracy of the pressure gradient term and divergence term could especially affect the high-frequency modes of acoustic and gravity waves. The high-frequency modes seem to be less significant meteorologically. We choose the second-order spatial scheme for terms of the pressure gradient and divergence terms to increase $\Delta t$ in spite of the decreasing accuracy of the terms, and the fourth-order scheme for the other terms.

\section{Appendix C: Density current experiment}

Straka et al. (1993) proposed a standard benchmark test of a non-linear density current problem, and we performed a test experiment with the same settings. A cold bubble whose size is $4 \times 2 \mathrm{~km}$ (horizontal and vertical, respectively) and minimum thermal perturbation is $-15 \mathrm{~K}$, is placed in the domain's center horizontally, and at $3 \mathrm{~km}$ height in a basic resting state whose potential temperature is constant at $300 \mathrm{~K}$. The domain is two-dimensional, and its size is $51.2 \mathrm{~km} \times 6.4 \mathrm{~km}$. Runs are done with various spatial resolutions of $200,100,50$, and $25 \mathrm{~m}$. The viscosity and diffusion are given by a coefficient of $75 \mathrm{~m}^{2} \mathrm{~s}^{-1}$ for the velocities and scalar quantities. 


\section{potential temp.}

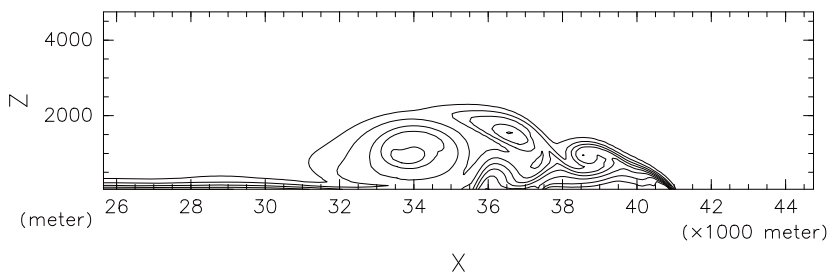

Figure C1. Plot of potential temperature at $t=900 \mathrm{~s}$ of the $100 \mathrm{~m}$ resolution density current experiment. Contour interval is $1 \mathrm{~K}$, and contours are centered around $300 \mathrm{~K}$.

Figure $\mathrm{C} 1$ shows a plot of the potential temperature at $t=900 \mathrm{~s}$ for the $100 \mathrm{~m}$ resolution run. The structure, such as the position of the front of the density current, rolls caused by the $\mathrm{K}-\mathrm{H}$ instability, and the magnitude at the local maxima, is reasonably similar with results of various models shown in Straka et al. (1993). The dependency of the $L_{2}$ norm of the potential temperature perturbation as a reference solution of the $25 \mathrm{~m}$ run of spatial resolution shows higher-order convergence than the second order between 100 and $50 \mathrm{~m}$ runs (Fig. C2). This model uses a fourth-order central difference scheme for advection, which has fourth-order accuracy with constant velocity, and second-order central difference scheme for the acoustic wave. The higher than second-order convergence seems to be due to the fourth-order difference scheme, although the velocity is not constant, and the scheme does not have fourth-order accuracy. The convergence is almost first-order between the 200 and $100 \mathrm{~m}$ runs. As Straka et al. (1993) mentioned, $200 \mathrm{~m}$ is too coarse to resolved this density current.

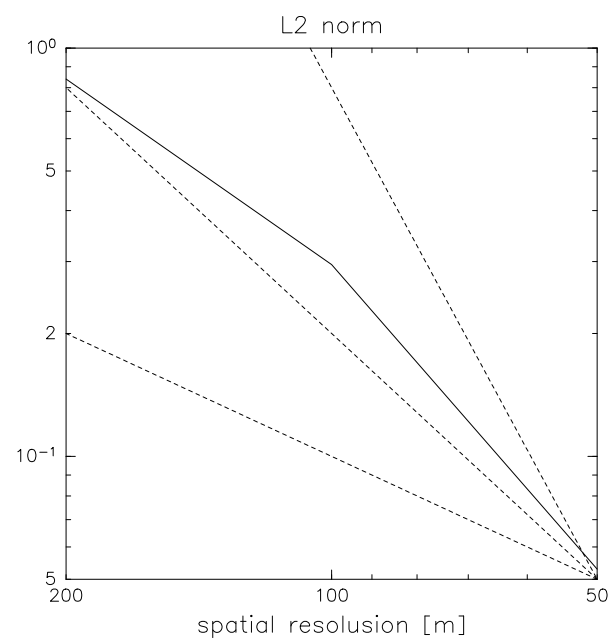

Figure C2. $L_{2}$ norm of potential temperature computed against the $25 \mathrm{~m}$ resolution experiment. Dashed lines represent first-, second-, and fourth-order convergence. 
Acknowledgements. The authors would like to thank the editor and the two referees, E. Goodfriend and an anonymous referee, for fruitful discussions. This model was developed by an interdisciplinary team at RIKEN Advanced Institute for Computational Science (AICS) called Team SCALE, from the fields of meteorological science and computer science. Part of the results in this paper was obtained using the $\mathrm{K}$ computer at the RIKEN AICS. Figures in this paper were drawn using tools developed by GFD-Dennou Club (http://www.gfd-dennou.org/index.html.en) and gnuplot (http://www.gnuplot.info/).

Edited by: P. Ullrich

\section{References}

Beljaars, A. C. M. and Holtslag, A. A. M.: Flux parameterization over land surfaces for atmospheric models, J. Appl. Meteorol., 30, 327-341, 1991.

Black, T. L.: The new NMC mesoscale Eta model: Description and forecast examples, Weather Forecast., 9, 265-278, 1994.

Brown, A. R., Derbyshire, S. H., and Mason, P. J.: Large-eddy simulation of stable atmospheric boundary layers with a revised stochastic subgrid model, Q. J. Roy. Meteor. Soc., 120, 14851512, 1994.

Deardorff, J. W.: On the magnitude of the subgrid scale eddy coefficient, J. Comput. Phys., 7, 120-133, 1971.

Deardorff, J. W.: Stratocumulus-capped mixed layers derived from a three-dimensional model, Bound.-Lay. Meteorol., 18, 495-527, 1980.

Favre, A.: Turbulence: space-time statistical properties and behavior in supersonic flows, Phys. Fluids, 26, 2851-2863, 1983.

Germano, M., Piomelli, U., Moin, P., and Cabot, W. H.: A dynamic subgridscale eddy viscosity model, Phys. Fluids A-Fluid, 3, 1760-1765, 1991 .

Gross, E. S., Bonaventura, L., and Rosatti, G.: Consistency with continuity in conservative advection schemes for free-surface models, Int. J. Numer. Meth. Fl., 38, 307-327, 2002.

Hodur, R. H.: The naval research laboratory's coupled ocean/atmosphere mesoscale prediction system (COAMPS), Mon. Weather Rev., 125, 1414-1430, 1997.

Horiuti, K.: A proper velocity scale for modeling subgrid-scale eddy viscosities in large eddy simulation, Phys. Fluids, 5, 146-157, 1993.

Ito, J., Tanaka, R., and Niino, H.: Large eddy simulation of dust devils in a diurnally-evolving convective mixed layer, J. Meteorol. Soc. Jpn., 88, 63-77, 2010.

Kawamura, T. and Kuwahara, K.: Computation of high Reynolds number flow around a circular cylinder with surface roughness, AIAA Paper, 84-0340, 1984.

Kessler, E.: On the distribution and continuity of water substance in atmospheric circulation, Meteor. Mon., 10, 1-84, 1969.

Klemp, J. B. and Wilhelmson, R. B.: The simulation of threedimensional convective storm dynamics, J. Atmos. Sci., 35, 1070-1096, 1978.

Kusaka, H. and Kimura, F.: Coupling a single-layer urban canopy model with a simple atmospheric model: Impact on urban heat island simulation for an idealized case, J. Meteorol. Soc. Jpn., 82, 67-80, 2004.
Kusaka, H., Kondo, H., Kikegawa, Y., and Kimura, F.: A simple single-layer urban canopy model for atmospheric models: Comparison with multi-layer and slab models, Bound.-Lay. Meteorol., 101, 329-358, 2001.

Lilly, D. K.: On the numerical simulation of buoyant convection, Tellus, 14, 148-171, 1962.

Lilly, D. K.: The representation of small-scale turbulence in numerical simulation experiments, in: Proceedings of the IBM scientific computing symposium on environmental sciences, White Plains, New York, IBM Form no. 320-1951, 195-209, 1967.

Louis, J.-F.: A parametric model of vertical eddy fluxes in the atmosphere, Bound.-Lay. Meteorol., 17, 187-202, 1979.

Mellor, G. L. and Yamada, T.: Development of a turbulence closure model for geophysical fluid problems, Rev. Geophys. Space Ge., 20, 851-875, 1982.

Miyamoto, Y., Kajikawa, Y., Yoshida, R., Yamaura, T., Yashiro, H., and Tomita, H.: Deep moist atmospheric convection in a subkilometer global simulation, Geophys. Res. Lett., 40, 4922-4926, 2013.

Miyamoto, Y., Yoshida, R., Yamaura, T., Yashiro, H., Tomita, H., and Kajikawa, Y.: Does convection vary in different cloudy disturbances?, Atmos. Sci. Lett., 16, 305-309, doi:10.1002/asl2.558, 2015.

Moeng, C.-H. and Wyngaard, J. C.: Spectral analysis of large-eddy simulations of the convective boundary layer, J. Atmos. Sci., 45 , 3573-3587, 1988.

Moin, P., Squires, K., Cabot, W., and Lee, S.: A dynamic subgridscale model for compressible turbulence and scalar transport, Phys. Fluids A-Fluid, 3, 2746-2757, 1991.

Nakanishi, M. and Niino, H.: An improved Mellor-Yamada level3 model with condensation physics: Its design and verification, Bound.-Lay. Meteorol., 112, 1-31, 2004.

Niwa, Y., Tomita, H., Satoh, M., and Imasu, R.: A threedimensional icosahedral grid advection scheme preserving monotonicity and consistency with continuity for atmospheric tracer transport, J. Meteorol. Soc. Jpn., 89, 255-268, 2011.

Saito, K., Fujita, T., Yamada, Y., Ishida, J., Kumagai, Y., Aranami, K., Ohmori, S., Nagasawa, R., Kumagai, S., Muroi, C., Kato, T., Eito, H., and Yamazaki, Y.: The operational JMA nonhydrostatic mesoscale model, Mon. Weather Rev., 134, 12661298, 2006.

Sato, Y., Nishizawa, S., Yashiro, H., Miyamoto, Y., Kajikawa, Y., and Tomita, H.: Impacts of cloud microphysics on trade wind cumulus: which cloud microphysics processes contribute to the diversity in a large eddy simulation?, Prog. Earth Planet. Sc., 2, 23, doi:10.1186/s40645-015-0053-6, 2015.

Satoh, M., Tomita, H., Yashiro, H., Miura, H., Kodama, C., Seiki, T., Noda, A. T., Yamada, Y., Goto, D., Sawada, M., Miyoshi, T., Niwa, Y., Hara, M., Ohno, Y., Iga, S., Arakawa, T., Inoue, T., and Kubokawa, H.: The non-hydrostatic icosahedral atmospheric model: description and development, PEPS, 1, 18, doi:10.1186/s40645-014-0018-1, 2014.

Seiki, T. and Nakajima, T.: Aerosol effect of the condensational process on a convective cloud simulation, J. Atmos. Sci., 71, 833853, 2014.

Sekiguchi, M. and Nakajima, T.: A k-distribution-based radiation code and its computational optimization for an atmospheric general circulation model, J. Quant. Spectrosc. Ra., 109, 2779-2793, 2008. 
Scotti, A., Meneveau, C., and Lilly, D. K.: Generalized Smagorinsky model for anisotropic grids, Phys. Fluids A-Fluid, 5, 23062308, 1993.

Skamarock, W.: Evaluating mesoscale NWP models using kinetic energy spectra, Mon. Weather Rev., 132, 3019-3032, 2004.

Skamarock, W. C., Klemp, J. B., Dudhia, J., Gill, D. O., Barker, D. M., Duda, M. G., Huang, X.-Y., Wang, W., and Powers, J. G.: A description of the Advanced Research WRF version 3, NCAR Technical Note, 475, 2008.

Smagorinsky, J.: General circulation experiments with the primitive equations, Mon. Weather Rev., 91, 99-164, 1963.

Straka, J. M., Wilhelmoson, R. B., Wicher, L. J., Anderson, J. R., and Droegemeier, K. K.: Numerical solutions of non-linear density current: A benchmark solution and comparisons, Int. J. Numer. Meth. Fl., 17, 1-22, 1993.

Sullivan, P. P. and Patton, E. G.: The effect of mesh resolution on convective boundary layer statistics and structures generated by large-eddy simulation, J. Atmos. Sci., 68, 2395-02415, 2011.

Sullivan, P. P., McWilliams, J. C., and Moeng, C.-H.: A subgridscale model for large-eddy simulation of planetary boundarylayer flows, Bound.-Lay. Meteorol., 71, 247-276, 1994.

Suzuki, K., Nakajima, T., Nakajima, T. Y., and Khain, A. P.: A study of microphysical mechanisms for correlation patterns between droplet radius and optical thickness of warm clouds with a spectral bin microphysics model, J. Atmos. Sci., 67, 1126-1141, 2010.

Tomita, H.: New microphysical schemes with five and six categories by diagnostic generation of cloud ice, J. Meteorol. Soc. Jpn., 86, 121-142, 2008.

Tomita, H. and Satoh, M.: A new dynamical framework of nonhydrostatic global model using the icosahedral grid, Fluid Dyn. Res., 34, 357-400, 2004.
Tsuboki, K. and Sakakibara, A.: Large-scale parallel computing of cloud resolving storm simulator, in: High Performance Computing, edited by: Zima, H. P., Joe, K., Sato, M., Seo, Y., and Shimasaki, M., Springer, 243-259, 2002.

Tripoli, G. J. and Cotton, W. R.: The Colorado State University three-dimensional cloud/mesoscale model-1982: Part I. General theoretical framework and sensitivity experiments, Atmos. Res., 16, 185-220, 1982.

Uno, I., Cai, X.-M., Steyn, D. G., and Emori, S.: A simple extension of the Louis method for rough surface layer modeling, Bound.Lay. Meteorol., 76, 395-409, 1995.

Wilson, D. K.: An alternative function for the wind and temperature gradients in unstable surface layers, Bound.-Lay. Meteorol., 99, 151-158, 2001.

Xue, M., Droegemeier, K. K., and Wong, V.: The advanced regional prediction system (ARPS) - a multiscale nonhydrostatic atmospheric simulation and prediction tool. Part I: Model dynamics and verification, Meteorol. Atmos. Physics., 75, 161-193, 2000.

Yakhot, A., Orszag, S., Yakhot, V., and Israeli, M.: Renormalization group formulation of large-eddy simulations, J. Sci. Comput., 4, 139-158, 1989.

Zalesk, S. T.: Fully multi-dimensional flux-corrected transport algorithms for fluids, J. Comput. Phys., 31, 335-362, 1979.

vanZanten, M. C., Stevens, B., Nuijens, L., Siebesma, A. P., Acherman, A., Burnet, F., Cheng, A., Couvreux, F., Jiang, H., Khairoutdinv, M., Kogan, Y., Lewellen, D. C., Mechem, D., Nakamura, K., Noda, A., Shipway, B. J., Slawinska, J., Wang, S., and Wyszogrodzki, A.: Controls on precipitation and cloudiness in simulations of trade-wind cumulus as observed during RICO, J. Adv. Model. Earth Syst., 3, M06001, doi:10.1029/2011MS000056, 2011. 